\title{
Particle optics of quadrupole doublet magnets in Spallation Neutron Source accumulator ring
}

\author{
J. G. Wang* \\ Oak Ridge National Laboratory, Oak Ridge, Tennessee 37831, USA
}

(Received 17 July 2006; published 21 December 2006)

\begin{abstract}
The Spallation Neutron Source ring employs doublet quadrupoles and dipole correctors in its straight sections. The electromagnetic quadrupoles have a large aperture, small aspect ratio, and relatively short iron-to-iron distance. The corrector is even closer to one of the quads. There have been concerns on the magnetic fringe field and interference in the doublet magnets and their assemblies. We have performed 3D computing simulations to study magnetic field distributions in the doublet magnets. Further, we have analyzed the particle optics based on the $z$-dependent focusing functions of the quads. The effect of the magnetic fringe field and interference, including the third-order aberrations, on the particle motion are investigated. The lens parameters and the first-order hard edge models are derived and compared with the parameters used in the ring lattice calculations.
\end{abstract}

DOI: 10.1103/PhysRevSTAB.9.122401

PACS numbers: 03.50.-z, 29.27.Eg, 41.85.Lc, 85.70.-w

\section{INTRODUCTION}

In the Spallation Neutron Source (SNS) just completed at Oak Ridge National Laboratory (ORNL), a $1 \mathrm{GeV} \mathrm{H}^{-}$ beam from its linac is injected into an accumulator ring to produce a proton beam, which is accumulated to high intensity before it strikes a target for the generation of intense neutrons. The SNS accumulator ring, designed and developed at Brookhaven National Lab (BNL) [1], contains four straight sections. Each section employs two quadrupole doublet assemblies for beam focusing. There are three magnets in each assembly: two quadrupoles and one dipole corrector $[2,3]$. They are designated as 30Q58, $30 \mathrm{Q} 44$, and 41CD30, where the first number is the magnet aperture in centimeters, and the second number is the magnet steel length in centimeters. The aspect ratio of the quads, defined as the steel length over the aperture, is only 1.93 and 1.47 , respectively, which is much smaller than that for most high energy accelerator magnets. The assembly is densely packed. The iron-to-iron distance between the two quads is $51.4 \mathrm{~cm}$, while it is only $21.4 \mathrm{~cm}$ between 30Q58 and 41CD30.

The small aspect ratio of the quads leads to significant fringe field. The relatively small iron-to-iron distance within the assembly causes magnetic interference between the two quads, as well as between 30Q58 and its neighboring corrector $41 \mathrm{CD} 30$. We have been concerned with the effect of the magnetic fringe field and interference in the SNS ring. Although the magnetic fringe field in quadrupoles has been well investigated [4-12], it appears that its effect is often overlooked in practice. This is reasonable for high energy accelerator magnets, in which the fringe field is insignificant; but it may not be the case for the magnets in low energy, high current accelerators such as the SNS. As far as the magnetic interference within magnet assemblies is concerned, to the best of our knowledge, there have

*Electronic address: jgwang@ornl.gov been no rigorous analyses of the subject in the published literature.

During the SNS ring development and construction, there have been neither measurements of the magnetic fringe field of an individual quad nor magnetic measurements of the quadrupole doublet assemblies. Thus, 3D computing simulations become the only way to address the issue [13]. We first simulate the doublet magnets in various models and to obtain their field distributions. The magnetic interference in the ring quadrupole doublet can then be expressed in terms of the changes in the integrated gradient and harmonics. We then employ the technique of 3D multipole expansion to derive the focusing functions in the models. The particle optics containing the effect of magnetic fringe field and interference can be computed precisely for the linear transfer matrices, lens parameters, equivalent hard edge models, nonlinear aberrations, etc. Though the analyses of the magnetic fringe field and interference presented and developed in this paper are based on simulation data, the techniques equally apply to measured data in experiments.

In Sec. II, we present 3D computing simulations of the quadrupole doublet magnets. The integrated field parameters such as the integrated gradient and harmonic contents are summarized. The $z$-dependent focusing functions are generated through 3D multipole expansion. Section III is devoted to the particle optics in a single $30 \mathrm{Q} 58$ and a single 30Q44. The fringe field is analyzed by directly solving the particle trajectory equations. The lens parameters are calculated and the correct hard edge models are derived, which are found to differ from conventional hard edge approximations. In Sec. IV, we develop a technique to analyze the magnetic interference between two magnets as a first-order perturbation. An example is demonstrated with the particle optics in 30Q58 plus 41CD30. It is shown that a symmetric hard edge model for the quad no longer exists due to the magnetic interference, which breaks down the magnetic field symmetry with respect to its center. 
Section V contains all the information on the two quads plus a corrector. The effect of magnetic fringe field and interference on particle optics is analyzed. The linear transfer matrices and correct hard edge models are obtained and compared with conventional treatments. The particle trajectories are verified by the OPERA3D TRACK command calculations. The nonlinear effect caused by the pseudomultipoles is discussed in Appendix A, where the thirdorder aberrations are demonstrated for individual quads and their assembly. A short summary of this work can be found in Sec. VI.

\section{3D COMPUTING SIMULATIONS OF QUAD DOUBLET}

\section{A. Simulation models}

The simulation environment employed in this study is OPERA3D/TOSCA [14]. We have built five different simulation models: a single $30 \mathrm{Q} 58$, a single $30 \mathrm{Q} 44$, $30 \mathrm{Q} 58$ plus 41CD30, 30Q58 plus 30Q44, and the quadrupole doublet assembly (two quads plus a corrector). The simulation models are built by the OPERA package "Modeller" rather than the "Pre-Processor." The Modeller makes it easier to simulate two or more magnets together. In building the models, special attention is paid to keep the mesh sizes of each magnet the same in the different models in order to avoid artificial interferences in calculations. The mechanical dimensions of the doublet magnets are taken from the BNL final design drawings and parameters. In the models, 30Q58 is energized at $810 \mathrm{~A}$ as a focusing quad for the $x$-motion, while $30 \mathrm{Q} 44$ is driven by $860 \mathrm{~A}$ as a defocusing quad for the $x$-motion. These currents should be close to the operation values for a $1 \mathrm{GeV}$ proton beam. The current of 30Q44 in one of the models is also varied to $960 \mathrm{~A}$ in order to see the dynamic effect of the interference. The corrector 41CD30 is represented by its steel core only. In the models with two or three magnets, the distance be- tween the two quad centers is $1.02489 \mathrm{~m}$, while the distance between $30 \mathrm{Q} 58$ center and 41CD30 center is $0.654304 \mathrm{~m}$. The fifth simulation model, which contains all three magnets, is shown in Fig. 1, where the central magnet is $30 \mathrm{Q} 58$, the right one is $30 \mathrm{Q} 44$, and the left iron core is for $41 \mathrm{CD} 30$.

\section{B. Two-dimensional field parameters}

In postprocessing of OPERA3D/TOSCA simulations, we first obtain a two-dimensional field representation for each model in the following form [15]:

$$
\begin{aligned}
& B_{r}(r, \theta)=\sum_{m=1}^{\infty} C_{m}\left(\frac{r}{R_{\text {ref }}}\right)^{m-1} \sin \left[m\left(\theta-\alpha_{m}\right)\right], \\
& B_{\theta}(r, \theta)=\sum_{m=1}^{\infty} C_{m}\left(\frac{r}{R_{\text {ref }}}\right)^{m-1} \cos \left[m\left(\theta-\alpha_{m}\right)\right] .
\end{aligned}
$$

Here $C_{m}$ and $\alpha_{m}$ are the amplitude and phase angle of the $2 \mathrm{~m}$-pole component of the total field, and $R_{\text {ref }}$ is a reference radius within the magnet aperture. This can be done by measuring the integrated field with a rotating Cartesian patch, similar to an unbucked winding in a Halbach search coil in accelerator magnet measurements [16]. For quadrupoles, the integrated gradient $\left(G^{*} L\right)$ and integrated harmonics $\left(C_{m}{ }^{*} L\right)$ are calculated. It is a common practice to express the normalized coefficients $N C_{m}$ of the integrated harmonic contents as

$$
N C_{m}=\frac{\left(C_{m}{ }^{*} L\right)}{\left(C_{2}{ }^{*} L\right)} \times 10^{4}=\frac{\left(C_{m}{ }^{*} L\right)}{\left(G^{*} L\right) R_{\text {ref }}} \times 10^{4} \text { (unit) }
$$

The results from different simulation models are listed in Table I. In the calculation, the reference radius $R_{\text {ref }}$ is $10 \mathrm{~cm}$. The reduction of the integrated gradient of 30Q58 due to its neighbors can reach more than $1 \%$. This effect is probably significant, considering the accuracy of the magnetic field requirement for the SNS ring is at the lower $10^{-4}$

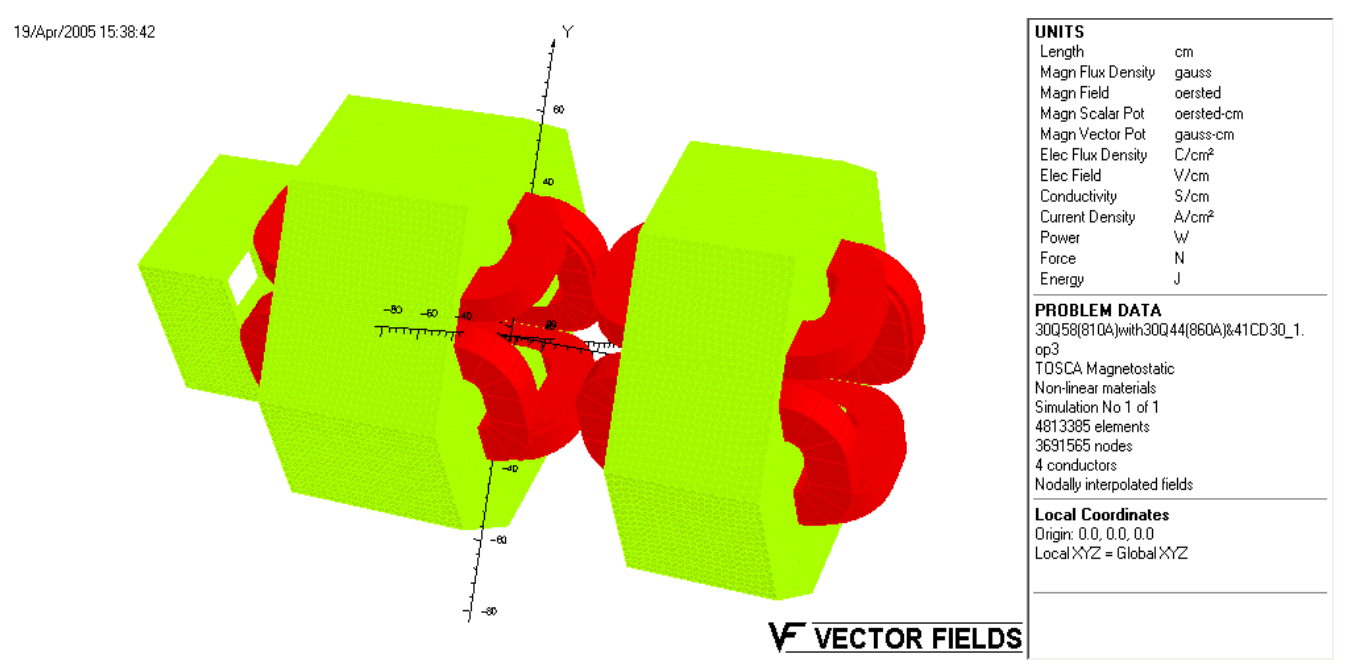

FIG. 1. (Color) Simulation model for SNS ring quadrupole doublet assembly (from left to right: 41CD30 iron core, 30Q58, 30Q44). 
TABLE I. Integrated gradients and harmonics in various models.

\begin{tabular}{llccccc}
\hline \hline & & $\begin{array}{c}G^{*} L \\
(\mathrm{~T})\end{array}$ & $\begin{array}{c}\Delta G^{*} L \\
(\%)\end{array}$ & $\begin{array}{c}\mathrm{NC}_{6} \\
(\text { Unit }\end{array}$ & $\begin{array}{c}\mathrm{NC}_{10} \\
\text { (Unit) }\end{array}$ & $\begin{array}{c}\mathrm{NC}_{14} \\
(\text { Unit) }\end{array}$ \\
\hline 30Q58 (810A) & Alone & 2.4576 & $100 \%$ & 2.43 & 7.62 & 0.94 \\
& w/41CD30 & 2.4488 & $99.64 \%$ & 2.63 & 7.42 & 0.94 \\
& w/30Q44 (860A) & 2.4427 & $99.40 \%$ & 3.83 & 7.55 & 0.95 \\
& w/30Q44 (960A) & 2.4421 & $99.37 \%$ & 4.07 & 7.62 & 0.99 \\
& w/30Q44 (860A) \& 41CD30 & 2.4325 & $98.98 \%$ & 3.25 & 7.76 & 0.99 \\
& w/30Q44 (960A) \& 41CD30 & 2.4319 & $98.95 \%$ & 3.39 & 7.62 & 0.98 \\
\hline 30Q44 (860A) & 2.0713 & $100 \%$ & 1.97 & 8.01 & 0.90 \\
& Alone & 2.0548 & $99.21 \%$ & 3.01 & 7.99 & 0.91 \\
& w/30Q58 (810A) & 2.0545 & $99.19 \%$ & 3.02 & 8.02 & 0.92 \\
\hline \hline
\end{tabular}

level. The dynamic effect of the interference, i.e., the effect on 30Q58 due to the current variation in 30Q44, seems negligible. The harmonic contents also change, but less than 2 units. The two-dimensional field parameters indicate the strength of magnetic interference. But, they can neither produce particle optics nor provide any information on the magnetic fringe field.

\section{3D multipole expansion}

The $z$-dependent field distributions, especially the linear focusing functions of the quads, can be obtained by the technique of 3D multipole expansion of the field data in simulations $[17,18]$. The expansion yields the generalized gradients based on the simulated radial field $B_{r}(R, \theta, z)$ on a cylindrical surface of radius $R$, which is coaxial with the magnet $z$-axis. The linear focusing function $k(z)$ of a quad is related to the normal, on-axis linear gradient $C_{2, s}(z)$ by

$$
k(z)=\frac{2 C_{2, s}(z)}{B_{\rho}}
$$

where $B_{\rho}$ is the magnetic rigidity of particles, which is $5.6574 \mathrm{Tm}$ for proton at $1 \mathrm{GeV}$. The calculations of the

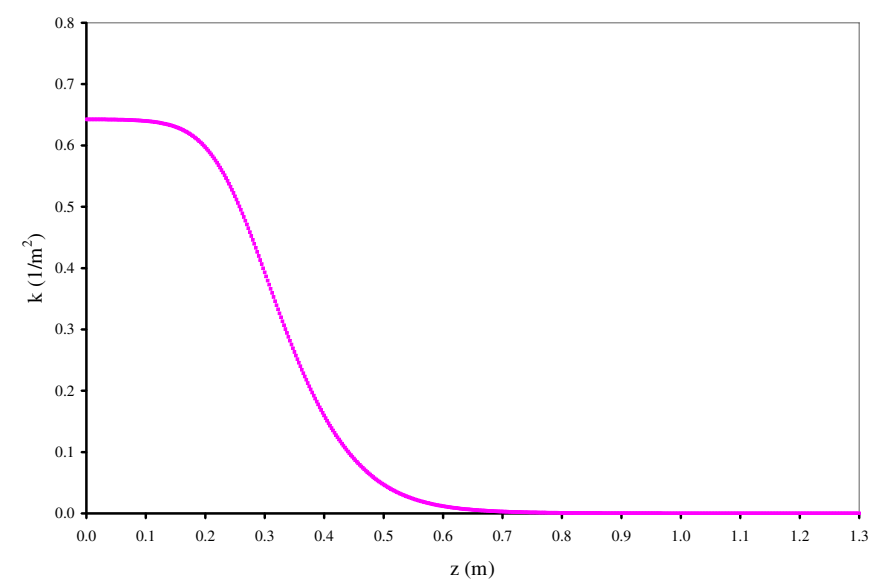

FIG. 2. (Color) Linear focusing function $k(z)$ of a single $30 \mathrm{Q} 58$. linear focusing functions have been performed for all the five simulation models.

The right half of the linear focusing function of a single 30Q58 from this analysis is plotted in Fig. 2. The reflection with respect to $z=0$ is implied. We cut off the plot at $z=$ $1.3 \mathrm{~m}$, beyond which the very small residual field is neglected. The average $k(z)$ in the central region from $z=0$ to $z=0.1 \mathrm{~m}$ is $0.64231 \mathrm{~m}^{-2}$, corresponding to a gradient of $3.6338 \mathrm{~T} / \mathrm{m}$. The integration of $k(z)$ from $z=0$ to $1.3 \mathrm{~m}$ yields $0.21738 \mathrm{~m}^{-1}$, which corresponds to an integrated gradient of $1.2298 \mathrm{~T}$.

\section{MAGNETIC FRINGE FIELD IN A SINGLE QUAD}

The optics of charged particles in magnetic quadrupoles has been well studied and the general information on the subject can be found in many textbooks [19-24]. In addition, many articles are specifically devoted to the analyses of magnetic fringe field in a single quad [4-11]. Although the theory on magnetic fringe field in a quadrupole has been well developed, it appears that its effects on particle optics are often overlooked in practice. Quads with small aspect ratios are often simply represented by "conventional hard edge models" obtained by calculating its magnetic length from the integrated gradient divided by the gradient around the magnet center. This in general is inaccurate for quads with significant fringe field, as shown for an "all fringe" quad in a more recent paper by Bernal et al. [12].

In this section we apply the existing fringe field theory to the first-order particle optics in a single $30 \mathrm{Q} 58$ and a single $30 \mathrm{Q} 44$, and compare the results with the conventional treatment. Based on the linear focusing functions obtained from the simulation data, the magnetic fringe field is analyzed with the direct solutions of the particle trajectory equations, leading to the linear transfer matrices of the quads. The hard edge models, as well as the lens parameters, are correctly derived from the matrix elements. A brief summary details the discrepancies between the firstorder hard edge models and the conventional hard edge 
models. The third-order effect, which comes from the fringe field such as the pseudo-octupole, is demonstrated by the rays on two symmetry planes and is included in Appendix A.

\section{A. Linear transfer matrices of 30 Q58}

The linear focusing function $k(z)$ of a single 30Q58, as shown in Fig. 2, already contains all the information about its magnetic fringe field. The particle motion in a quad with such a focusing function is dictated by the linear trajectory equations expressed by

$$
\begin{aligned}
& x^{\prime \prime}+k(z) x=0, \\
& y^{\prime \prime}-k(z) y=0 .
\end{aligned}
$$

With the program MATHEMATICA [25], we solve numerically the second-order differential equations by two firstorder differential equations of the following kind:

$$
\begin{aligned}
& u_{1}^{\prime}=u_{2}, \\
& u_{2}^{\prime}= \pm k(z) u_{1},
\end{aligned}
$$

where $u$ represents either $x$ or $y$, and $\left(u_{1}, u_{2}\right)$ forms a pair of phase space parameters. In numerical solutions we employ two sets of initial conditions. A ray with $u_{1}=1$ and $u_{2}=$ 0 at the initial position $z_{1}=-1.3 \mathrm{~m}$ yields the matrix components of $M_{11}(z)$ and $M_{21}(z)$, while a ray with $u_{1}=$ 0 and $u_{2}=1$ leads to the matrix elements of $M_{12}(z)$ and $M_{22}(z)$. The four matrix elements for the $x$-motion are plotted in Fig. 3. These curves are also the principle ray traces. The linear transfer matrix $\mathbf{M}_{x}$ of a focusing 30Q58 from $z_{1}=-1.3 \mathrm{~m}$ to $z_{2}=1.3 \mathrm{~m}$ is evaluated from these traces as

$$
\mathbf{M}_{x}=\left[\begin{array}{cc}
0.461063 & 1.91587 \\
-0.410998 & 0.461063
\end{array}\right] .
$$

For the $y$-motion in 30Q58, the four linear transfer matrix elements or ray traces are plotted in Fig. 4. The linear

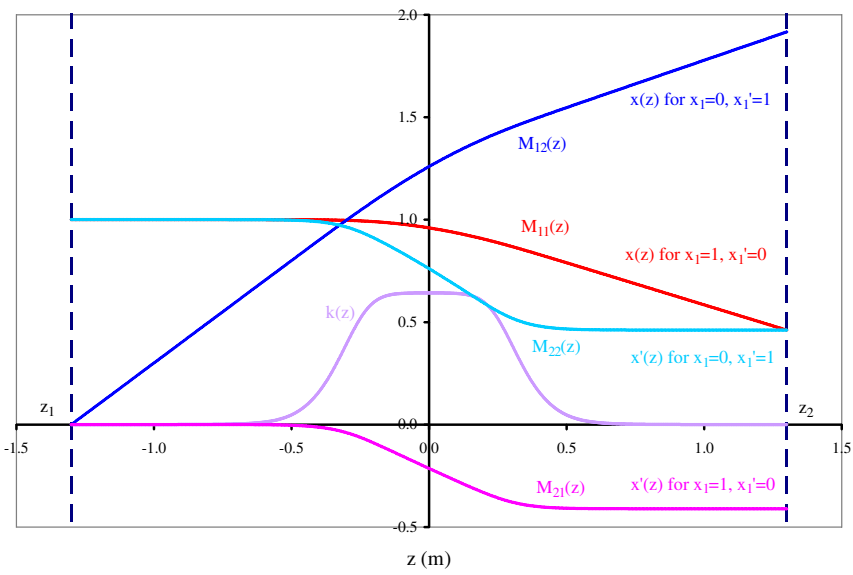

FIG. 3. (Color) Matrix elements and ray traces for the $x$-motion in 30Q58.

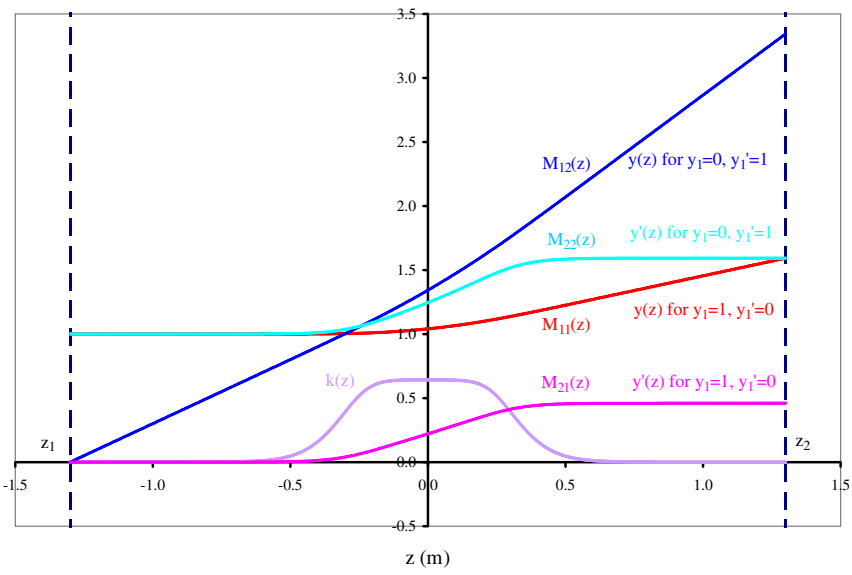

FIG. 4. (Color) Matrix elements and ray traces for the $y$-motion in 30Q58.

transfer matrix from $z_{1}=-1.3 \mathrm{~m}$ to $z_{2}=1.3 \mathrm{~m}$ is given as

$$
\mathbf{M}_{y}=\left[\begin{array}{cc}
1.59235 & 3.34248 \\
0.459412 & 1.59235
\end{array}\right] .
$$

The numerical values of the determinant of these two matrices are very, very close to unity, indicating they are symplectic.

\section{B. Lens parameters and hard edge models}

With the linear transfer matrices in (6), the four lens parameters of the quad can be obtained. The focusing length is simply expressed as

$$
f=f_{1}=f_{2}=-\frac{1}{M_{21}}
$$

and the principle plane distance is

$$
\begin{aligned}
& d_{1}=-\frac{1-M_{22}}{M_{21}}-l_{1}, \\
& d_{2}=-\frac{1-M_{11}}{M_{21}}-l_{2} .
\end{aligned}
$$

Here $l_{1}$ and $l_{2}$ are the distances from the lens center to the entrance and exit, respectively. For a symmetric lens such as a single 30Q58, $l_{1}=l_{2}=l$ and $M_{22}=M_{11}$, thus, $d_{1}=$ $d_{2}=d$. The numerical solutions of the 30Q58 lens parameters are listed in Table II.

A symmetric lens has only two independent parameters $(f, d)$. A symmetric hard edge model also has two independent parameters $\left(L, k_{0}\right)$. Therefore, it is possible to have an equivalent hard edge model for each symmetric lens. The equivalence is valid only in the sense that the hard edge model should yield the same transfer matrix from the lens entrance to exit. Thus, we require the following relationship:

$$
\mathbf{M}=\mathbf{D H D} .
$$


TABLE II. 30Q58 hard edge models and lens parameters.

\begin{tabular}{lccc}
\hline \hline & Conventional & $\begin{array}{c}\text { 1st-order } \\
\text { focusing }\end{array}$ & $\begin{array}{c}\text { 1st-order } \\
\text { defocusing }\end{array}$ \\
\hline$L(\mathrm{~m})$ & 0.6769 & 0.7757 & 0.7794 \\
$k_{0}\left(1 / \mathrm{m}^{2}\right)$ & 0.6423 & 0.5609 & 0.5575 \\
$k_{0}{ }^{*} L(1 / \mathrm{m})$ & 0.4348 & 0.4351 & 0.4345 \\
$\varphi($ degree $)$ & 31.08 & 33.28 & 33.34 \\
$d(\mathrm{~cm})$ & & 1.129 & 1.064 \\
$f_{x}(\mathrm{~m})$ & 2.417 & 2.433 & \\
$f_{y}(\mathrm{~m})$ & -2.191 & & -2.177 \\
\hline \hline
\end{tabular}

Here $\mathbf{M}$ is the transfer matrix of 30Q58 found above, and

$$
\mathbf{D}=\left[\begin{array}{cc}
1 & l-L / 2 \\
0 & 1
\end{array}\right]
$$

is the transfer matrix for a drift distance of $(l-L / 2)$. The transfer matrix for a hard edge model is

$$
\mathbf{H}=\left[\begin{array}{cc}
C & S / \kappa \\
\mp \kappa S & C
\end{array}\right],
$$

where $C$ and $S$ are $\cos (\varphi)$ and $\sin (\varphi)$ for a focusing quad, $\cosh (\varphi)$ and $\sinh (\varphi)$ for a defocusing quad, respectively, $\varphi$ is the effective lens focusing strength, and kappa, $\kappa$, is the square root of the constant focusing function $k_{0}$. The upper sign (minus) in $\mathrm{H}_{21}$ is for focusing, while the lower sign (plus) for defocusing. This relationship leads to a transcendental equation for $\varphi[6,19]$ :

$$
M_{11}-M_{21} l=C \pm \frac{1}{2} \varphi S .
$$

The other hard edge parameters can be found as

$$
\begin{gathered}
\kappa=\frac{M_{21}}{\mp S}, \\
L=\frac{\varphi}{\kappa} .
\end{gathered}
$$

Equation (10a) can be solved numerically to obtain the

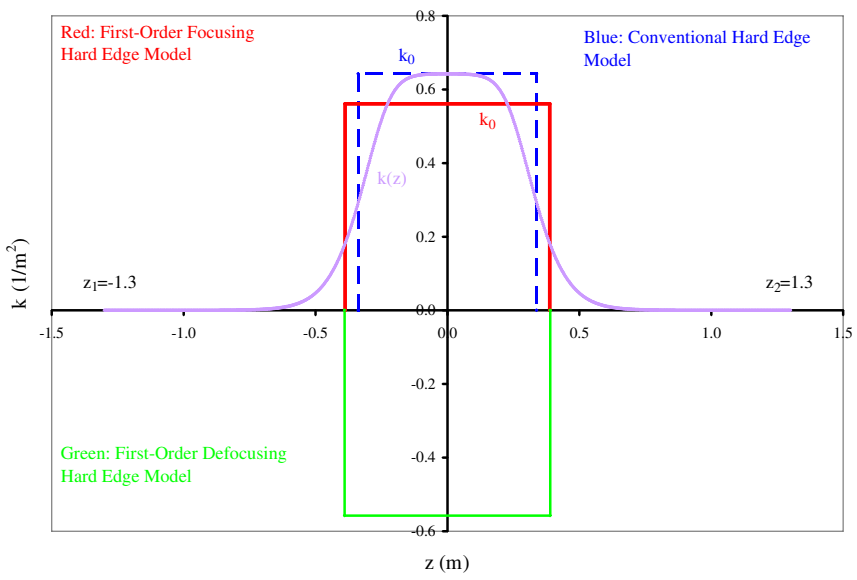

FIG. 5. (Color) Hard edge models for 30Q58. effective lens strength $\varphi$, and further to get $\kappa, k_{0}$, and $L$ from Eqs. (10b) and (10c). The hard edge parameters thus calculated for 30 Q58 are also listed in Table II.

The various hard edge models for a single 30Q58 are plotted in Fig. 5. The linear focusing function $k(z)$ is also included. In the "conventional hard edge model," its height equals the central strength of the quad, and its effective length is calculated from the integrated gradient divided by the gradient around the quad center. The firstorder hard edge models come from rigorous computations based on the first-order trajectory equations and the resulting linear transfer matrices.

\section{Particle optics in a single 30 Q44}

The linear focusing function $k(z)$ of a single 30Q44 obtained from the simulation and 3D multipole expansion is shown in Fig. 6, where the reflection with respect to $z=$ 0 is implied. The computation of particle optics in $30 \mathrm{Q} 44$ follows the same procedure as for 30Q58. We first calculate the linear transfer matrices according to Eq. (5). The lens parameters and the hard edge models are then computed. We skip the detailed steps and plots, and only list the numerical results in Table III.

In a brief summary, the plot in Fig. 5 and the numerical data in Tables II and III show clearly the differences between the conventional hard edge model and the firstorder hard edge models. First, the hard edge parameters $k_{0}$, $L$, and $k_{0}{ }^{*} L$ in the first-order focusing and defocusing models are slightly different. Thus, we need in principle two different hard edge models for focusing and defocusing, rather than one in the conventional treatment $[6,19]$. This may be very inconvenient in applying these models in the lattice design codes such as TRANSPORT [26] and MAD [27]. For many applications, we may still use one firstorder hard edge model with the mean values of $k_{0}$ and $L$ between the focusing and defocusing models as an approximation. Second, the hard edge height is no longer equal to that at the quad center, as in the case of the conventional model. In 30Q58, the mean hard edge height

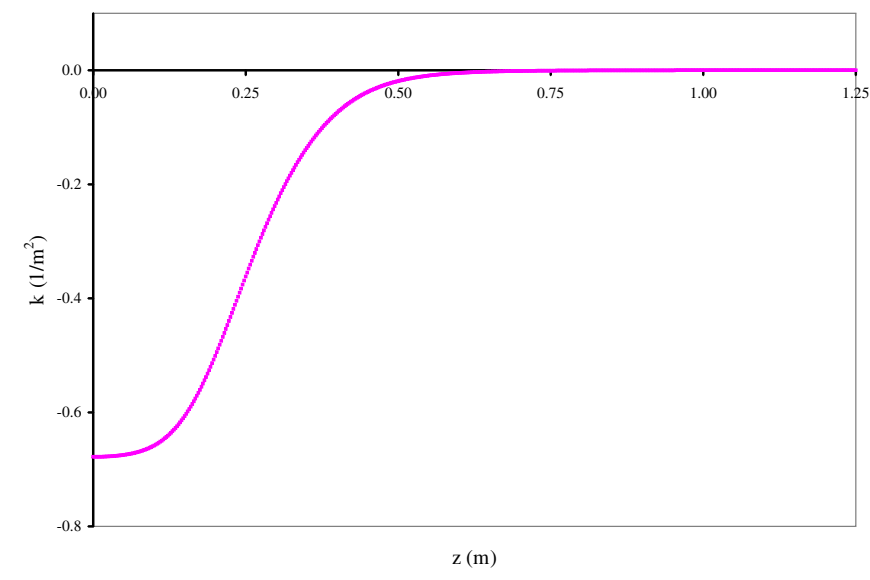

FIG. 6. (Color) Linear focusing function of a single 30Q44. 
TABLE III. 30Q44 hard edge models, lens parameters, and matrix elements.

\begin{tabular}{lccc}
\hline \hline & Conventional & $\begin{array}{c}\text { 1st-order } \\
\text { focusing }\end{array}$ & $\begin{array}{c}\text { 1st-order } \\
\text { defocusing }\end{array}$ \\
\hline$L(\mathrm{~m})$ & 0.5407 & 0.6605 & 0.6635 \\
$k_{0}\left(1 / \mathrm{m}^{2}\right)$ & -0.6770 & 0.5547 & -0.5513 \\
$k_{0}{ }^{*} L(1 / \mathrm{m})$ & -0.3661 & 0.3664 & -0.3658 \\
$\varphi($ degree $)$ & 25.49 & 28.18 & 28.23 \\
$d(\mathrm{~cm})$ & & 0.683 & 0.655 \\
$f_{x}(\mathrm{~m})$ & 2.824 & 2.843 & \\
$f_{y}(\mathrm{~m})$ & -2.644 & & -2.626 \\
Transfer matrix elements from $z_{1}=-1.25 \mathrm{~m}$ to & $z_{2}=1.25 \mathrm{~m}$ \\
$M_{11}$ & & 0.5579 & 1.4735 \\
$M_{12}(\mathrm{~m})$ & & 1.9580 & 3.0757 \\
$M_{21}(1 / \mathrm{m})$ & & -0.3518 & 0.3808 \\
$M_{22}$ & & 0.5579 & 1.4735 \\
\hline \hline
\end{tabular}

$k_{0}$ is about $15 \%$ smaller than that in the conventional model, while it is about $22 \%$ for $30 \mathrm{Q} 44$. Accordingly, the mean hard edge length $L$ is longer than that in the conventional model by roughly the same amount. Third, the product of the hard edge height and length is not quite the same as the integrated focusing function. The relationship is held only for the geometric mean value between the first-order focusing and defocusing models [6]. Fourth, as a result of all the above differences, the lens parameters also differ from that in the conventional hard edge model. For instance, the effective lens strength $\varphi$ in the first-order hard edge models of $30 \mathrm{Q} 58$ and $30 \mathrm{Q} 44$ is $2.2^{\circ}$ to $2.7^{\circ}$ more than that in the conventional hard edge model, which would result in different focusing lengths and tunes. The focusing lengths of $30 \mathrm{Q} 58$ and $30 \mathrm{Q} 44$ predicted by the first-order hard edge models differ from that of the conventional model by about $0.7 \%$. The particle optics will further depart from the prediction of the conventional hard edge model by magnetic interference as described below.

\section{MAGNETIC INTERFERENCE BETWEEN TWO MAGNETS}

Small particle accelerator rings usually are densely packed due to very limited spaces among lattice components. Magnetic field between adjacent magnets are often overlapped or affected by surrounding materials. That leads to magnetic interference, which may play an important role in machine commissioning and operation [28]. The study of magnetic interference has been limited so far to simulation or measurement of the changes in the integrated gradient and harmonics of a quad due to its neighbors [13,28-30]. These changes, such as the data in Table I, are a measure of the strength of magnetic interference. But, they neither provide any information about the effect on particle motion, nor can they be used to compensate the integrated strength of quads in an existing lattice consisting of conventional hard edge models. This has motivated us to develop more rigorous analyses of particle optics under the influence of magnetic interference in quadrupole assemblies.

In this section, we analyze the magnetic interference between a quad (30Q58) and a corrector (41CD30). The change in the linear focusing function of 30Q58 due to the presence of $41 \mathrm{CD} 30$ is first obtained from the two relevant simulation models. The effect on the beam optics due to the magnetic interference between 30 Q58 and 41CD30 can be computed as a first-order perturbation. The linear transfer matrices are obtained and correct hard edge models are constructed for a perturbed quad. The results are also compared with that from a conventional hard edge model. For the magnetic interference between two quads (30Q58 and 30Q44), the same analysis procedure applies, which is included in Sec. V.

\section{A. Change in linear focusing function}

When a magnetic corrector is present in the vicinity of a quadrupole, the quadrupole field distribution is changed. In general, the integrated gradient of the quadrupole is reduced since the quadrupole flux lines are terminated by the corrector iron core. As shown in Table I, the integrated gradient of 30Q58 plus 41CD30 steel is $2.4488 \mathrm{~T}$, compared to $2.4576 \mathrm{~T}$ when the corrector is absent. The relative change in the integrated gradient is $0.36 \%$. To further understand the effect on particle optics due to the magnetic interference, we first need to compute the linear focusing function of the model with 30Q58 and 41CD30. This is shown in Fig. 7, where $k(z)$ is the linear focusing function of $30 \mathrm{Q} 58$ plus $41 \mathrm{CD} 30$ and $k_{1}(z)$ is the one for $30 \mathrm{Q} 58$ alone. The two linear focusing functions with and without $41 \mathrm{CD} 30$ overlap very well in most of the region except around $z=-0.5 \mathrm{~m}$, where the corrector is located. This can be seen much more clearly in Fig. 8, where we plot the difference, $\delta k(z)=k(z)-k_{1}(z)$, in the linear focusing functions of $30 \mathrm{Q} 58$ with and without $41 \mathrm{CD} 30$. It is the

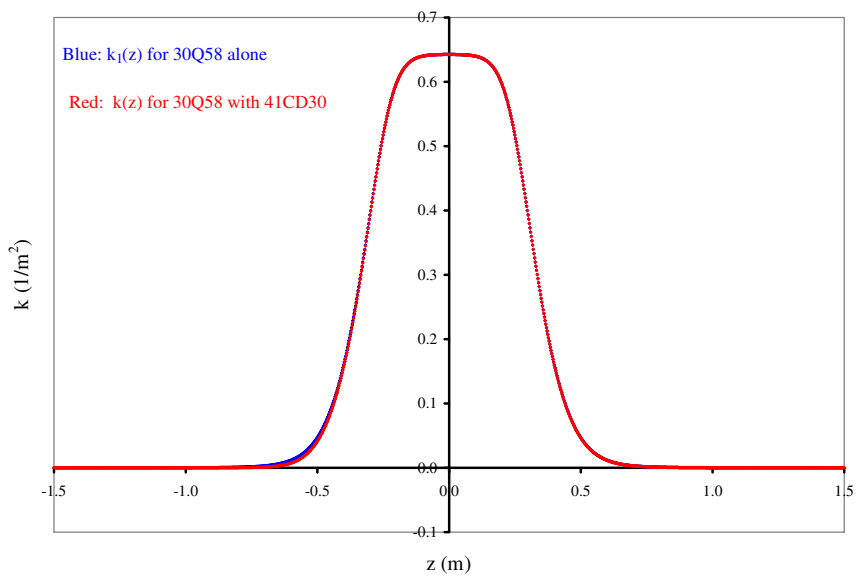

FIG. 7. (Color) Linear focusing functions for 30Q58 with and without 41CD30. 


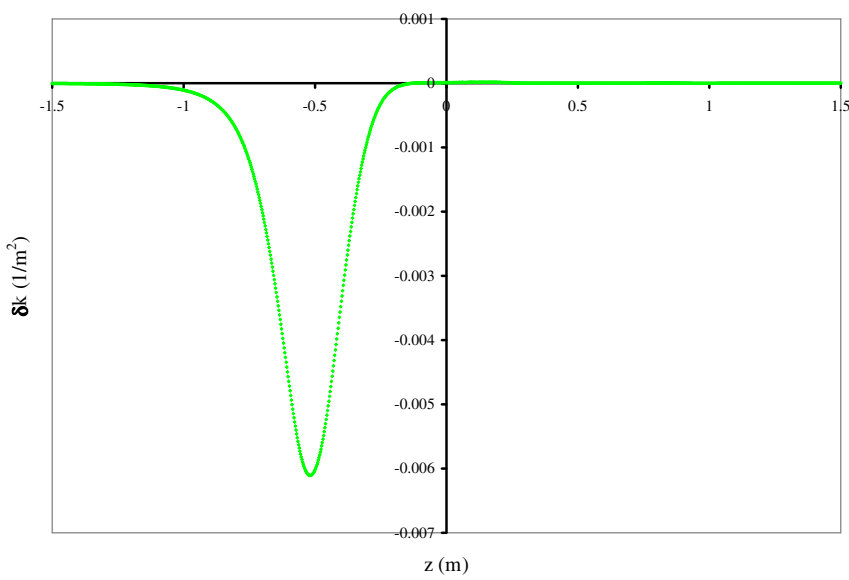

FIG. 8. (Color) Linear focusing function difference for $30 \mathrm{Q} 58$ with and without 41CD30.

change in this linear focusing function that determines the extent of the interference, which can be precisely computed.

\section{B. Magnetic interference as a first-order perturbation}

The integration of $\delta k(z)$ in Fig. 8 from $z_{1}=-1.3 \mathrm{~m}$ to $z_{2}=1.3 \mathrm{~m}$ is less than $1 \%$ of the $k_{1}(z)$ integration over the same region (see Table I). Thus, the magnetic interference between 30Q58 and 41CD30 can be treated as a first-order perturbation [31]. The equations of motion for $\delta x$ and $\delta y$, which are the changes in particle trajectories due to the perturbation, are thus expressed by

$$
\begin{aligned}
& \delta x^{\prime \prime}+k_{1}(z) \delta x=-\delta k(z) x, \\
& \delta y^{\prime \prime}-k_{1}(z) \delta y=\delta k(z) y .
\end{aligned}
$$

Here the linear focusing function $k_{1}(z)$ is the unperturbed parameter for $30 \mathrm{Q} 58$ alone, while $\delta k(z)$ is the perturbation shown in Fig. 8. The second-order terms $\delta k^{*} \delta x$ and $\delta k^{*} \delta y$ on the right-hand side (RHS) of Eq. (11) are neglected. The established procedure for solving Eq. (11) is to replace $x$ and $y$ on the right-hand side by the appropriate unperturbed solutions, i.e., to use $M_{11}(z)$ of the matrices (6a) and (6b) for the ray with $x_{1}=1$ and $x_{1}{ }^{\prime}=0$; and to use $M_{12}(z)$ for the ray with $x_{1}=0$ and $x_{1}{ }^{\prime}=1$, etc. The numerical solutions for $\delta \mathbf{x}(z)$ and $\delta \mathbf{y}(z)$ are plotted in Figs. 9 and 10, respectively. At the exit $z_{2}=1.3 \mathrm{~m}$, the changes in the linear transfer matrix of 30Q58 alone due to the perturbation of $41 \mathrm{CD} 30$ are for the $x$-motion

$$
\delta \mathbf{M}_{x}=\left[\begin{array}{ll}
2.846 & 2.141 \\
1.403 & 1.076
\end{array}\right] \times 10^{-3}
$$

and for the $y$-motion

$$
\delta \mathbf{M}_{y}=\left[\begin{array}{ll}
-3.888 & -2.892 \\
-2.264 & -1.700
\end{array}\right] \times 10^{-3} .
$$

Another approach for the interference problem is to solve Eq. (5) directly with the linear focusing function

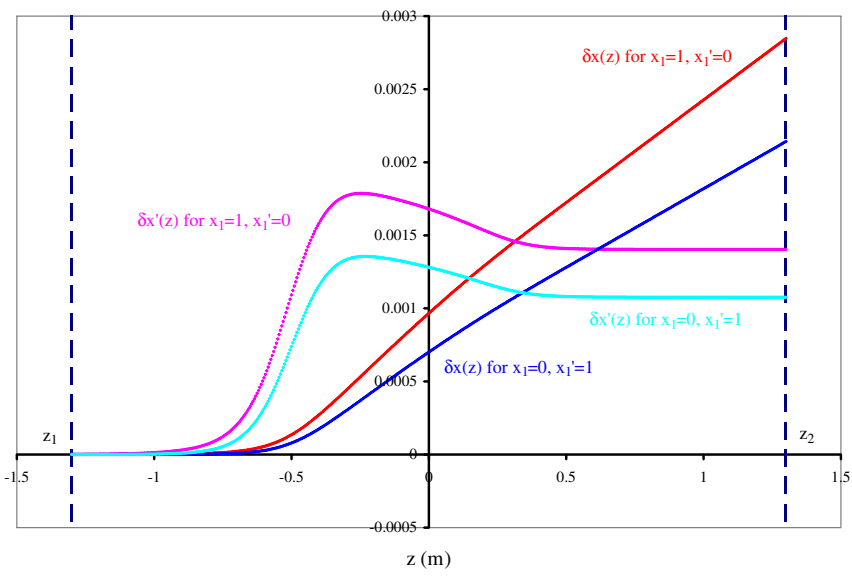

FIG. 9. (Color) Perturbations on particle trajectories for $x$-motion in 30Q58 plus 41CD30.

$k(z)$ in Fig. 7. We then find the transfer matrix from $z_{1}=$ $-1.3 \mathrm{~m}$ to $z_{2}=1.3 \mathrm{~m}$ for the $x$-motion in $30 \mathrm{Q} 58$ plus $41 \mathrm{CD} 30$ as

$$
\mathbf{M}_{x \mathbf{1}}=\left[\begin{array}{cc}
0.46391 & 1.91802 \\
-0.40960 & 0.46214
\end{array}\right] \text {. }
$$

For the $y$-motion in 30Q58 plus 41CD30, the linear transfer matrix is

$$
\mathbf{M}_{y \mathbf{1}}=\left[\begin{array}{ll}
1.58846 & 3.33959 \\
0.45715 & 1.59065
\end{array}\right]
$$

It is easy to verify numerically that the matrices in (13a) and (13b) are the sum of the matrices in (6a) and (6b) and (12a) and (12b), i.e. $\mathbf{M}_{x(y) \mathbf{1}}=\mathbf{M}_{x(y)}+\delta \mathbf{M}_{x(y)}$, as we expected.

\section{Hard edge models for a perturbed quad}

In the matrices of (13a) and (13b) the two diagonal elements are not equal, and the matrices now have three independent elements due to the asymmetry of the quad

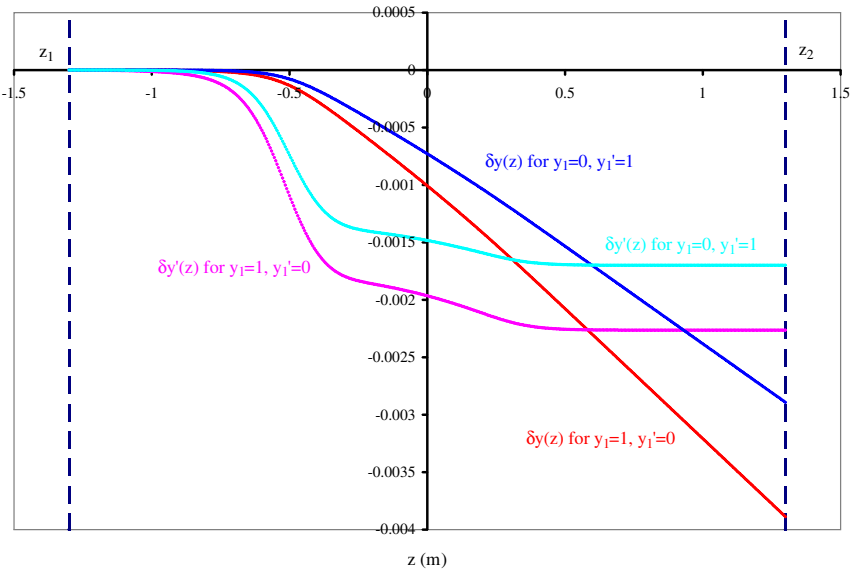

FIG. 10. (Color) Perturbations on particle trajectories for $y$-motion in 30Q58 plus 41CD30. 
perturbed by an adjacent corrector. A symmetric equivalent hard edge model as derived before does not exist any more, since that model has only two independent parameters.

There are a few options to derive hard edge models for asymmetric quads. For the first one, let us keep the hard edge models from the first-order matrices for a single 30Q58 unchanged, as shown in Fig. 5. The perturbation due to $41 \mathrm{CD} 30$ could be represented by another perturbation hard edge model starting at $z=-L / 2-w$ and ending at $z=-L / 2$. This is to require

$$
\mathbf{M}_{1}=\mathbf{D H H}_{1} \mathbf{D}_{1} \text {. }
$$

Here $\mathbf{M}_{1}$ is the perturbed matrices in (13a) and (13b), $\mathbf{D}$ and $\mathbf{H}$ are the matrices in (9) for the unperturbed quad, $\mathbf{H}_{1}$ is the desired perturbation hard edge model due to the interference, and $\mathbf{D}_{1}$ is a drift space matrix with a distance of $-L / 2-w-z_{1}$. By employing Eq. (9), we find a relation

$$
\mathbf{D}_{1}^{-1} \mathbf{H}_{1}^{-1}=\mathbf{M}_{1}^{-1} \mathbf{M D}^{-1} \equiv \mathbf{Q} .
$$

The $\mathbf{H}_{1}$ parameters can then be found as

$$
\begin{aligned}
& \varphi=\kappa w=C^{-1}\left(\mathbf{Q}_{22}\right), \\
& \kappa=\mp \mathbf{Q}_{21} / S .
\end{aligned}
$$

As before, $C$ and $S$ are cos and sin for focusing, and cosh and sinh for defocusing. The numerical values show that for the $x$-motion the perturbation hard edge model $\mathbf{H}_{1}$ has an effective lens strength $\varphi=1.36^{\circ}$, height $k_{0}=$ $-0.005859 \mathrm{~m}^{-2}$, and length $w=0.3101 \mathrm{~m}$. As we expected from $\delta k(z)$ polarity in Fig. $8, \mathbf{H}_{1}$ represents a defocusing effect for a focusing 30Q58. For the $y$-motion, the perturbation hard edge model $\mathbf{H}_{1}$ has an effective lens strength $\varphi=1.35^{\circ}$, height $k_{0}=$ $0.00595 \mathrm{~m}^{-2}$, and length $w=0.3058 \mathrm{~m}$. It is a focusing element prior to the unperturbed hard edge model $\mathbf{H}$. This is shown in Fig. 11, where the main hard edge models are the same as in Fig. 5 for a single 30Q58, and the height of the perturbation hard edge models are magnified by a factor of 10 for clarity. A conventional hard edge model for the perturbed 30Q58 is also plotted for the purpose of comparison. This model is still symmetric with respect to $z=0$ and its height $k_{0}$ and length $L$ are obtained in a conventional way as before.

For the second option, we may consider an equivalent hard edge model asymmetric with respect to the magnet center $(z=0)$ and sandwiched in between two drift spaces of unequal distances. This is to require

$$
\mathbf{M}_{1}=\mathbf{D}_{2} \mathbf{H D}_{1} .
$$

Here $\mathbf{D}_{1}$ is the drift matrix from $z_{1}$ to $-L_{1}$

$$
\mathbf{D}_{1}=\left[\begin{array}{cc}
1 & -L_{1}-z_{1} \\
0 & 1
\end{array}\right]
$$

and $\mathbf{D}_{2}$ is the drift matrix from $L_{2}$ to $z_{2}$,

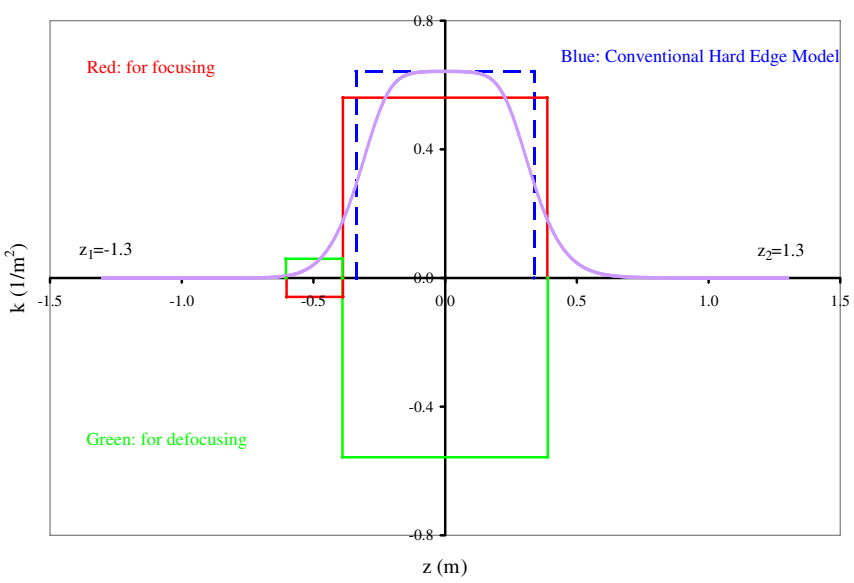

FIG. 11. (Color) Hard edge models for magnetic interference in $30 \mathrm{Q} 58$ plus 41CD30.

$$
\mathbf{D}_{2}=\left[\begin{array}{cc}
1 & z_{2}-L_{2} \\
0 & 1
\end{array}\right]
$$

and $\mathbf{H}$ is the transfer matrix for a hard edge quad of length $L=L_{1}+L_{2}$, as defined before. It can be shown that the effective lens strength $\varphi$ of the hard edge model can be found from the following transcendental equation with the matrix elements from the matrices $\mathbf{M}_{1}$ in (13a) and (13b)

$$
\frac{M_{11}+M_{22}}{2}-M_{21} z_{2}=C \pm \frac{1}{2} \varphi S
$$

Other hard edge parameters for $\mathbf{H}$ are obtained as

$$
\begin{gathered}
L_{1}=l-\frac{M_{22}-C}{M_{21}}, \\
L_{2}=l-\frac{M_{11}-C}{M_{21}}, \\
\kappa=\frac{M_{21}}{\mp S} .
\end{gathered}
$$

It is easy to see that if the quad is symmetric with respect to $z=0$, i.e., $M_{11}=M_{22}$, we recover the previous hard edge model with $L_{1}=L_{2}=L / 2$.

With the numerical values for the focusing and defocusing matrices, we compute the asymmetric hard edge models for 30 Q58 plus 41CD30. The resulting plot would be similar to that in Fig. 5, but with different parameters as listed in Table IV. In comparison with the data in Table II, we see that the perturbation from 41CD30 reduces the 30Q58 total hard edge length $L$ and the effective lens strength $\varphi$, and slightly increases its hard edge height $k_{0}$, in addition it makes the hard edge model asymmetric with respect to its center at $z=0$. 
TABLE IV. Hard edge models for 38Q58 plus 41CD30.

\begin{tabular}{lccc}
\hline \hline & Conventional & Focusing & Defocusing \\
\hline$-L_{1}(\mathrm{~m})$ & -0.3370 & -0.3815 & -0.3829 \\
$L_{2}(\mathrm{~m})$ & 0.3370 & 0.3858 & 0.3877 \\
$L(\mathrm{~m})$ & 0.6740 & 0.7673 & 0.7706 \\
$k_{0}\left(1 / \mathrm{m}^{2}\right)$ & 0.6423 & 0.5646 & 0.5615 \\
$k_{0}{ }^{*} L(1 / \mathrm{m})$ & 0.4329 & 0.4332 & 0.4327 \\
$\varphi($ degree $)$ & 30.95 & 33.03 & 33.09 \\
$f_{x}(\mathrm{~m})$ & 2.4261 & 2.4414 & \\
$f_{y}(\mathrm{~m})$ & -2.2011 & & -2.1875 \\
\hline \hline
\end{tabular}

\section{PARTICLE OPTICS IN QUAD DOUBLET ASSEMBLY}

In the SNS quadrupole doublet assembly as shown in Fig. 1, the two quadrupoles 30Q58 and 30Q44 interfere with each other, in addition to the interference between 30Q58 and 41CD30. The effect of the interference on particle optics can be analyzed in the same procedure as presented in Sect. IV. We first obtain the linear focusing function from the fifth simulation model. The changes in particle motion due to the interference can be obtained from the perturbed ray traces. We also construct hard edge models for the assembly, in which the identity of the two quads remains. The results are compared with the conventional model. The validity of the technique developed here is demonstrated by the agreement with the OPERA TRACK command calculations of the particle trajectories. The third-order aberrations in the assembly due to the magnetic fringe and interference are analyzed and included in Appendix A.

\section{A. Magnetic fringe and interference}

In Fig. 12 we plot three linear focusing functions: the blue curve $k_{1}(z)$ represents the linear focusing function of 30 Q58 alone, which is the same curve as in Fig. 2; the green curve $k_{2}(z)$ shows the linear focusing function of

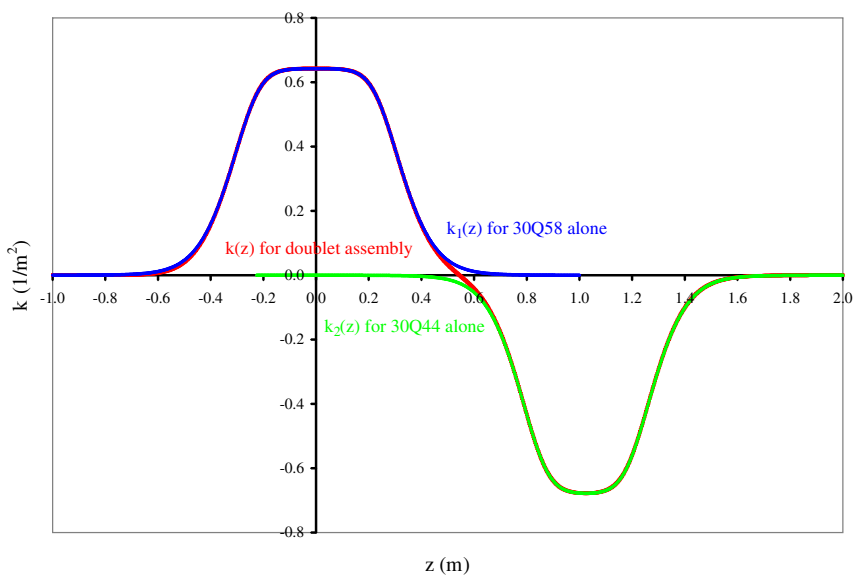

FIG. 12. (Color) Linear focusing functions in doublet assembly.

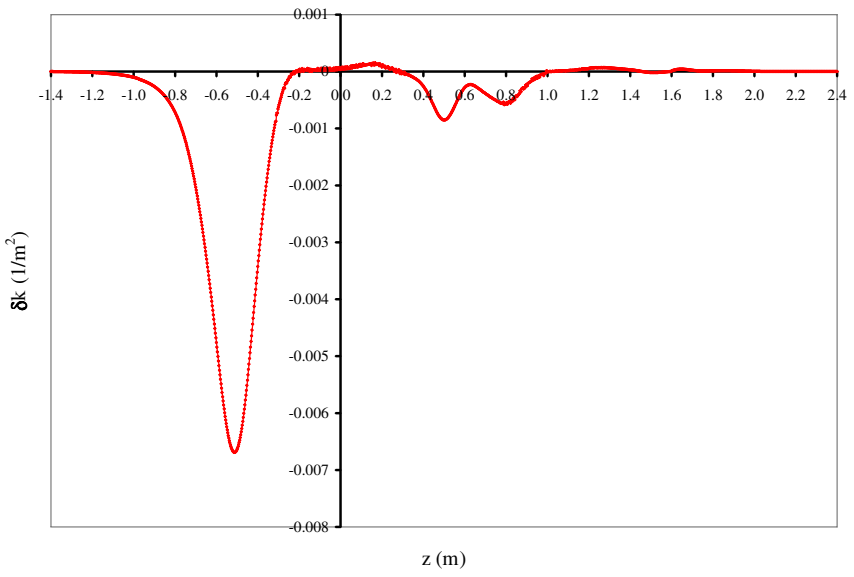

FIG. 13. (Color) Difference in $k(z)$ and $k_{1}(z)+k_{2}(z)$.

30Q44 itself, which is the same curve as in Fig. 6 but is offset by $z=1.02489 \mathrm{~m}$; the red curve $k(z)$ is the linear focusing function when the two quads and the corrector steel are together in simulation. The differences between $k(z)$ and $k_{1}(z)+k_{2}(z)$ appear around $z=0.5 \mathrm{~m}$ between the two quads, as well as around $z=-0.5 \mathrm{~m}$ due to magnetic interference from the corrector 41CD30. A better picture for this effect is shown in Fig. 13, where we plot the difference between $k(z)$ and $k_{1}(z)+k_{2}(z)$, i.e., $\delta k(z)=$ $k(z)-\left[k_{1}(z)+k_{2}(z)\right]$. It is obvious that the magnetic interference is dominated by the effect of 41CD30 on 30Q58.

We first use the linearly superimposed transfer function $k_{1}(z)+k_{2}(z)$ in Eq. (5) to obtain the unperturbed transfer matrices, which do not take into account the effect of the magnetic interference. Then, by employing $\delta k(z)$ in Fig. 13, we can solve Eq. (11) for the perturbations on the particle trajectories, as shown in Figs. 14 and 15. We evaluate these perturbations on the trajectories at an exit $z_{2}=2.275 \mathrm{~m}$. This results in the perturbation matrices from $z_{1}=-1.3 \mathrm{~m}$ to $z_{2}=2.275 \mathrm{~m}$ as

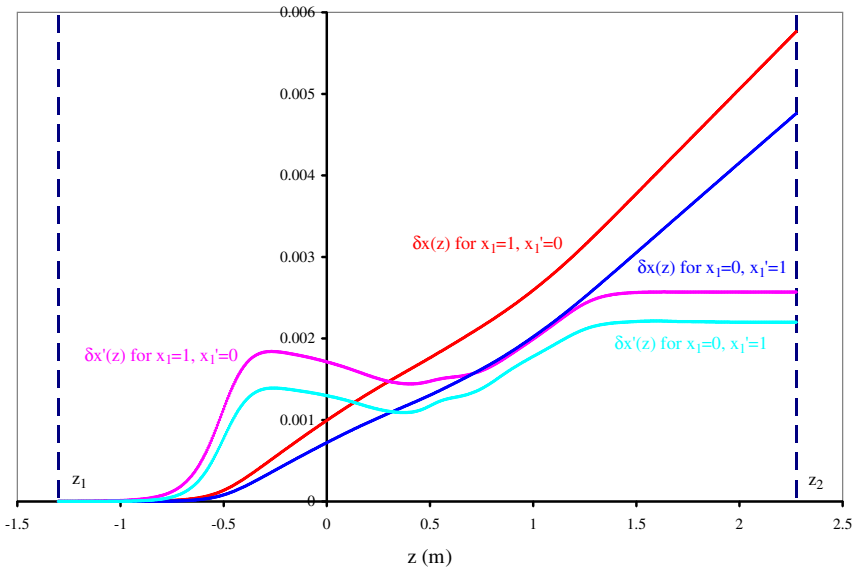

FIG. 14. (Color) Perturbations on $x$-motion due to magnetic interference in quad doublet assembly. 


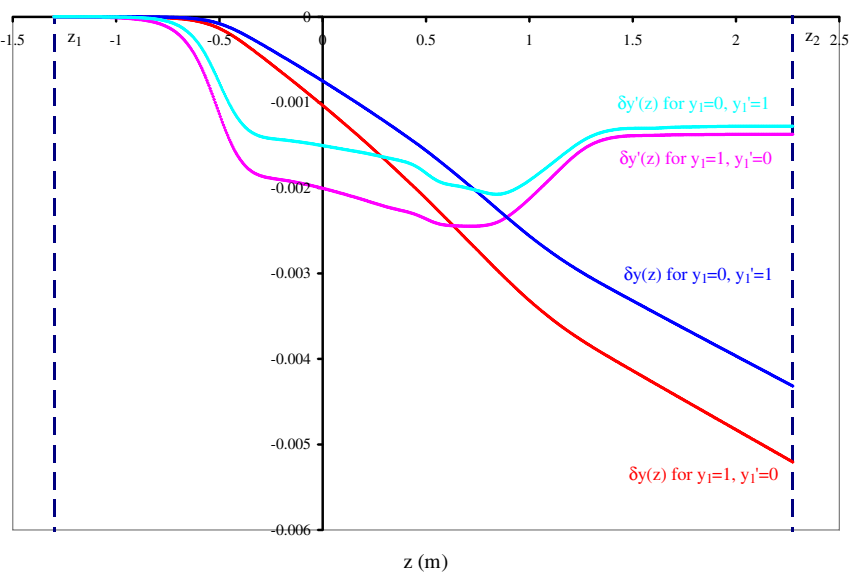

FIG. 15. (Color) Perturbations on $y$-motion due to magnetic interference in quad doublet assembly.

$$
\begin{aligned}
\delta \mathbf{M}_{x} & =\left[\begin{array}{ll}
5.73 & 4.63 \\
2.54 & 2.08
\end{array}\right] \times 10^{-3}, \\
\delta \mathbf{M}_{y} & =\left[\begin{array}{ll}
-5.12 & -4.14 \\
-1.29 & -1.11
\end{array}\right] \times 10^{-3} .
\end{aligned}
$$

As in the case of $30 \mathrm{Q} 58$ plus 41CD30, the differences between these unperturbed matrices and the perturbation matrices lead to the actual transfer matrices described in the next section, which include the effect of the magnetic interference in the assembly.

\section{B. Linear transfer matrices and hard edge models}

By employing the red curve $k(z)$ in Fig. 12, we can solve Eq. (5) directly to obtain the particle trajectories for the entire region of the quadrupole doublet assembly, as shown in Figs. 16 and 17. The linear transfer matrices for mapping particles from $z_{1}=-1.3 \mathrm{~m}$ to $z_{2}=2.275 \mathrm{~m}$ are then obtained as

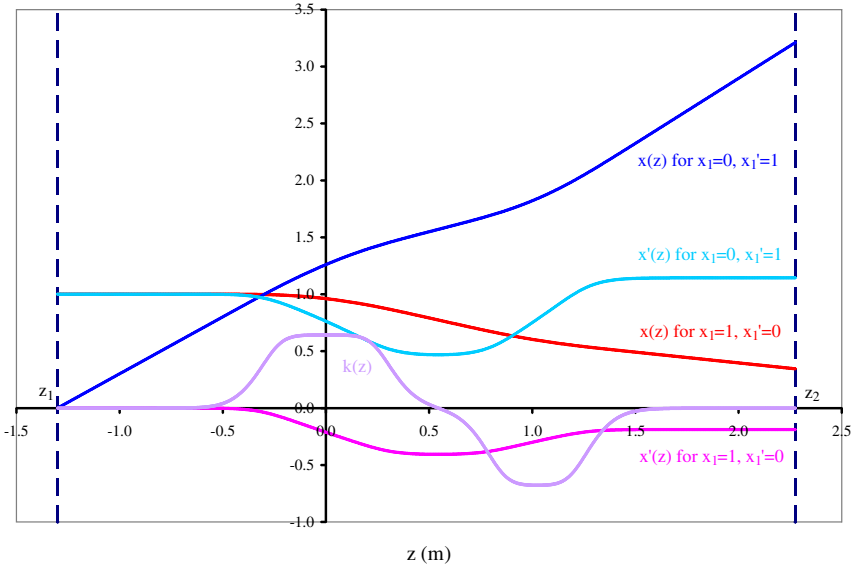

FIG. 16. (Color) Particle trajectories on the $x$-motion in the doublet assembly.

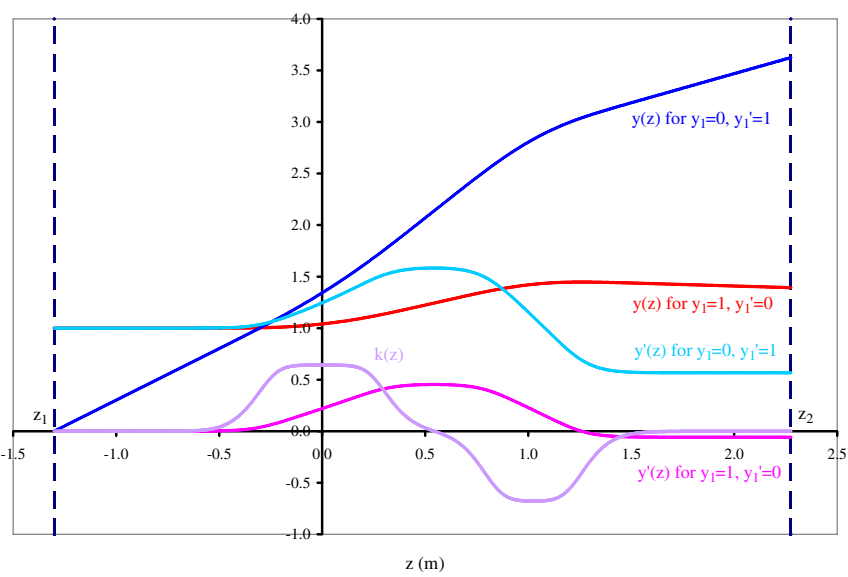

FIG. 17. (Color) Particle trajectories on the $y$-motion in the doublet assembly.

$$
\begin{aligned}
& \mathbf{M}_{x}=\left[\begin{array}{cc}
0.34458 & 3.2097 \\
-0.18881 & 1.1434
\end{array}\right], \\
& \mathbf{M}_{y}=\left[\begin{array}{cc}
1.3919 & 3.6236 \\
-0.058763 & 0.56549
\end{array}\right] .
\end{aligned}
$$

These matrices in principle suffice to represent the quad assembly in the lattice codes $[25,26]$. However, many accelerator designers are often accustomed with hard edge representations of magnets in lattices. In order to derive the hard edge models, in which the identity of the two quads still remain, we separate them at $z_{0}=$ $0.54455 \mathrm{~m}$, where the linear focusing function $k(z)$ crosses zero. By employing a similar approach as that for 30Q58 plus 41CD30 in Eqs. (17) and (18), we obtain the hard edge models for the quad doublet assembly as shown in Fig. 18. The hard edge parameters are listed in Table V.

We now compare three hard edge models for the quad doublet assembly. The first one is the correct hard edge model derived from the linear transfer matrices in (20). The second is that taking into account the fringe field of each

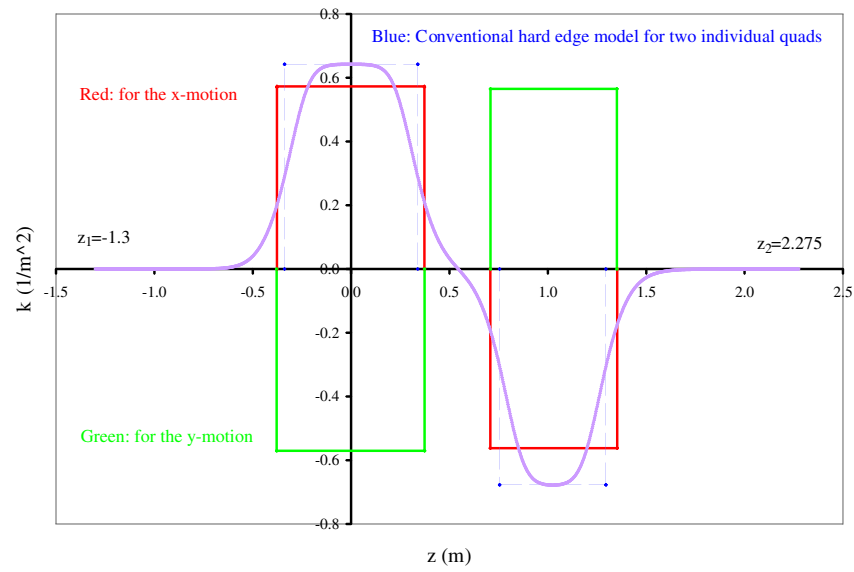

FIG. 18. (Color) Hard edge models for quad doublet assembly. 
TABLE V. Hard edge models and lens parameters for quad doublet assembly.

\begin{tabular}{|c|c|c|c|c|c|c|}
\hline & \multicolumn{2}{|c|}{ 1. First-order theory } & \multicolumn{2}{|c|}{ 2. No interference } & \multicolumn{2}{|c|}{ 3. Conventional } \\
\hline & $x$-motion & $y$-motion & $x$-motion & $y$-motion & $x$-motion & $y$-motion \\
\hline & \multicolumn{6}{|c|}{ Hard edge parameters } \\
\hline & \multicolumn{2}{|c|}{ 30Q58 } & \multicolumn{2}{|c|}{ 30Q58 } & \multicolumn{2}{|c|}{ 30Q58 } \\
\hline$k_{0}\left(1 / \mathrm{m}^{2}\right)$ & 0.5728 & -0.5704 & 0.5609 & -0.5575 & 0.6423 & -0.6423 \\
\hline$L_{1}(\mathrm{~m})$ & 0.3769 & 0.3785 & 0.3879 & 0.3897 & 0.3385 & 0.3385 \\
\hline$L_{2}(\mathrm{~m})$ & 0.3726 & 0.3736 & 0.3879 & 0.3897 & 0.3385 & 0.3385 \\
\hline \multirow[t]{2}{*}{$L(\mathrm{~m})$} & 0.7495 & 0.7521 & 0.7757 & 0.7794 & 0.6769 & 0.6769 \\
\hline & \multicolumn{2}{|c|}{ 30Q44 } & \multicolumn{2}{|c|}{ 30Q44 } & \multicolumn{2}{|c|}{ 30Q44 } \\
\hline$k_{0}\left(1 / \mathrm{m}^{2}\right)$ & -0.5624 & 0.5652 & -0.5513 & 0.5547 & -0.6770 & 0.6770 \\
\hline$L_{1}(\mathrm{~m})$ & 0.3170 & 0.3161 & 0.3318 & 0.3303 & 0.2704 & 0.2704 \\
\hline$L_{2}(\mathrm{~m})$ & 0.3274 & 0.3257 & 0.3318 & 0.3303 & 0.2704 & 0.2704 \\
\hline \multirow[t]{2}{*}{$L(\mathrm{~m})$} & 0.6443 & 0.6418 & 0.6635 & 0.6605 & 0.5407 & 0.5407 \\
\hline & \multicolumn{6}{|c|}{ Transfer matrix elements from $z_{1}=-1.3 \mathrm{~m}$ to $z_{2}=2.275 \mathrm{~m}$} \\
\hline$M_{11}$ & 0.34458 & 1.3919 & 0.33878 & 1.3971 & 0.32935 & 1.3874 \\
\hline$M_{12}(\mathrm{~m})$ & 3.2097 & 3.6236 & 3.2050 & 3.6278 & 3.1907 & 3.6148 \\
\hline$M_{21}(1 / \mathrm{m})$ & -0.18881 & -0.058763 & -0.19140 & -0.057356 & -0.19656 & -0.06275 \\
\hline \multirow[t]{2}{*}{$M_{22}$} & 1.1434 & 0.56549 & 1.1411 & 0.56685 & 1.1320 & 0.55726 \\
\hline & \multicolumn{6}{|c|}{ Lens pa } \\
\hline$f(\mathrm{~m})$ & 5.30 & 17.02 & 5.22 & 17.43 & 5.09 & 15.93 \\
\hline
\end{tabular}

individual quad, but neglecting all the interferences. The third one is from the conventional hard edge model for $30 \mathrm{Q} 58$ and 30Q44, as indicated by the blue dotted lines in Fig. 18. The hard edge parameters for the two quads in the second and third models are in Table II for 30Q58 and in Table III for 30Q44. The hard edge parameters, transfer matrix elements, and the lens parameters for the three models are listed in Table V. In these models, 30 Q58 center is at $z=0$, while it is at $z=1.02489 \mathrm{~m}$ for $30 \mathrm{Q} 44$. The two quads are sandwiched by three drift distances starting from $z_{1}=-1.3 \mathrm{~m}$ and ending at $z_{2}=2.275 \mathrm{~m}$. It can be seen that the discrepancies between the conventional hard edge model and the correct first-order model are quite significant. The focusing lengths $f_{x}$ and $f_{y}$ in the first-

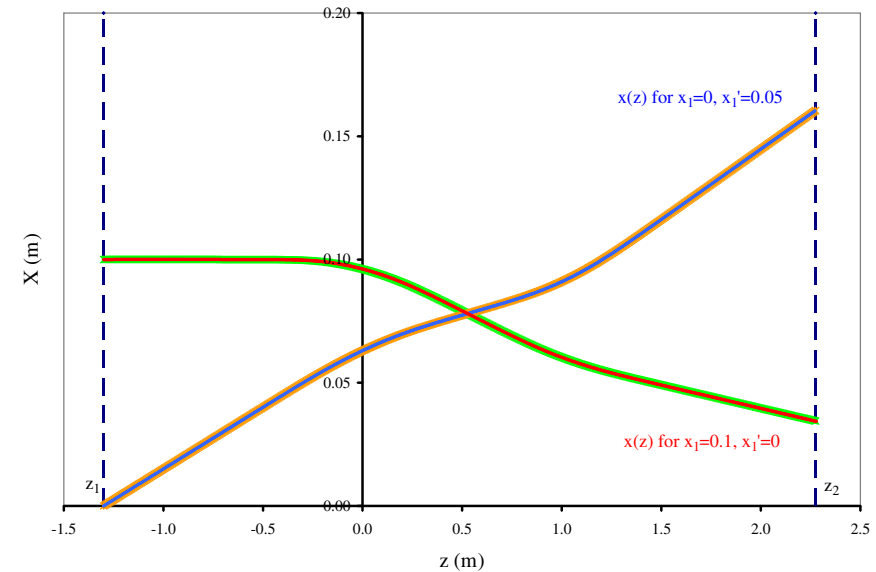

FIG. 19. (Color) Ray traces for $x$-motion from OPERA3D (blue and red) and matrix method. order model are 5.30 and $17.02 \mathrm{~m}$, respectively, while they are 5.09 and $15.93 \mathrm{~m}$ in the conventional model. The parameters in the second hard edge model are closer to the correct ones than the conventional hard edge model.

\section{Verification of particle trajectories}

OPERA3D has a built-in TRACK command for calculating trajectories of charged particles by directly integrating the equations of motion in simulated magnetic field. We launch a proton particle of $1 \mathrm{GeV}$ in energy at $z_{1}=$ $-1.3 \mathrm{~m}$ with different initial conditions: two rays on the $x$-plane with $x_{1}=0.1 \mathrm{~m}, x_{1}{ }^{\prime}=0$ and $x_{1}=0, x_{1}{ }^{\prime}=0.05$; two rays on the $y$-plane with $y_{1}=0.1 \mathrm{~m}, y_{1}{ }^{\prime}=0$ and $y_{1}=$ $0, y_{1}^{\prime}=0.05$. The code calculates the ray traces through

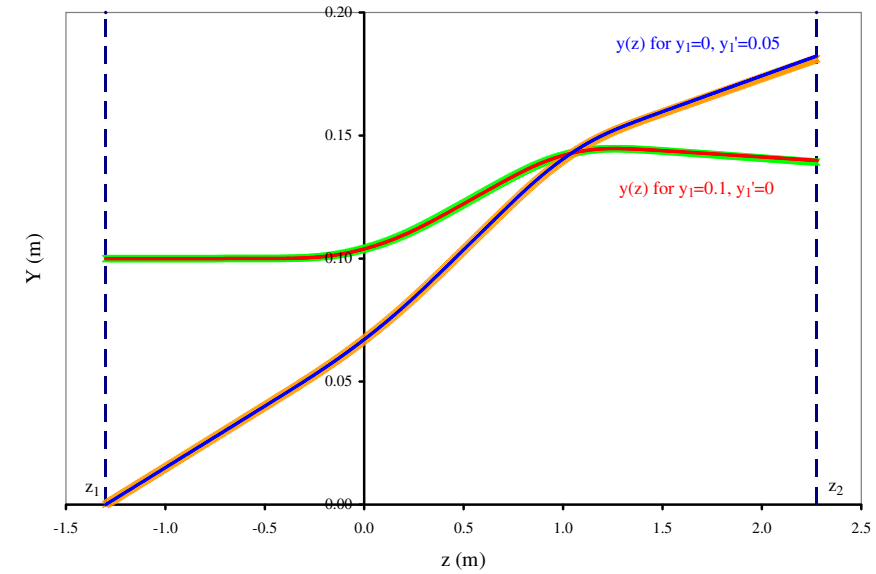

FIG. 20. (Color) Ray traces for $y$-motion from OPERA3D (blue and red) and matrix method. 
the dipole corrector and the quadrupole doublet. We plot the $x$-traces from OPERA3D in Fig. 19 and the $y$-traces in Fig. 20. In order to compare with the ray traces calculated from the matrix method, we overlay the ray trajectories from the first-order calculation in Figs. 16 and 17 scaled by the initial parameters, as shown by green and orange color. The agreement between the OPERA3D TRACK and the matrix method is excellent. Since the two methods are independent, this proves that the computation of particle optics with magnetic fringe field and interference developed above can be trusted.

\section{SUMMARY}

The particle optics in the SNS ring quadrupole doublet assemblies has been studied comprehensively based on their linear focusing functions resulted from 3D simulation and multipole expansion. These linear focusing functions contain all the necessary information on the magnetic fringe field and interference in the devices. The particle trajectories and linear transfer matrices are obtained for individual quads and their assemblies. The lens parameters are computed and the correct hard edge models for individual quad and their assemblies are constructed. The techniques presented and developed here equally apply to experimental data.

The magnetic fringe field in a quadrupole is analyzed by directly solving the equations of motion. The fringe field in a magnetic quad with small aspect ratio has a significant effect on the particle optics. The hard edge models resulted from the first-order transfer matrices depart from the conventional hard edge model in terms of their equivalent strength and effective length, etc. The third-order aberrations due to the pseudomultipoles in the fringe field are demonstrated with two rays on the symmetry planes. For the initial conditions used in calculations, the third-order aberrations change the particle parameters by up to a few percents in the quad assembly.

The magnetic interference in two or three magnets is investigated as a first-order perturbation. Its effect on the particle motion can be precisely computed from the changes in the linear focusing functions. In the SNS ring quadrupole doublet assemblies, the interference from $41 C D 30$ is dominant. The actual particle trajectories, the linear transfer matrices, and the lens parameters differ significantly from that produced by the conventional hard edge model. For instance, the focal lengths $f_{x}$ and $f_{y}$ are 5.32 and $17.02 \mathrm{~m}$, respectively, from the first-order theory, in comparison with $f_{x}=5.09 \mathrm{~m}$ and $f_{y}=15.93 \mathrm{~m}$ in the conventional hard edge model.

The differences in hard edge model parameters between our analyses and conventional ones, especially in the focusing length and effective lens strength of a quad doublet, suggest that we may want pay special attention to the critical focusing points such as injection foils and final focusing on target in the SNS operation. The SNS ring consists of many more magnet assemblies densely packed with other large aperture quadrupoles such as 21Q40 and 26Q40 in its arc regions, in addition to the quad doublet assemblies in its straight sections. The same problem of magnetic fringe field and interferences exists in these arc quad assemblies, and construction of correct hard edge models for these assemblies is in order. It is our on-going work to analyze these assemblies, following the technique we have developed for the doublet. Our plan is to provide correct magnet models for our lattice experts, who will have a solid foundation to reexamine the ring lattice calculations for the effect of magnetic fringe field and interferences in the SNS ring. This will be a subject of future work.

\section{ACKNOWLEDGMENTS}

ORNL/SNS is managed by UT-Battelle, LLC, for the U.S. Department of Energy under Contract No. DE-AC0500OR22725. The author would like to thank N. Tsoupas, who performed the physical design of the doublet quadrupoles and provided us with many useful comments on 3D simulations. We would also like to thank M. Venturini for his reading of this manuscript and his valuable comments on the subject.

\section{APPENDIX A: THIRD-ORDER ABERRATIONS IN SNS QUADRUPOLE DOUBLET}

For a reasonably well designed quadrupole in simulation, only the allowed terms $(m=2,6,14$, etc.) exist and their skew components can also be neglected. Thus, the quadrupole field ( $m=2$ only) can be obtained by the 3D multipole expansion as [17]

$$
\begin{aligned}
& B_{r}(r, z)=2 C_{2, s}(z) r-\frac{1}{3} C_{2, s}^{\prime \prime}(z) r^{3}+O(5), \\
& B_{\theta}(r, z)=2 C_{2, s}(z) r-\frac{1}{6} C_{2, s}{ }^{\prime \prime}(z) r^{3}+O(5), \\
& B_{z}(r, z)=2 C_{2, s}^{\prime}(z) r^{2}+O(4),
\end{aligned}
$$

The second terms in Eqs. (A1a) and (A1b) are so-called

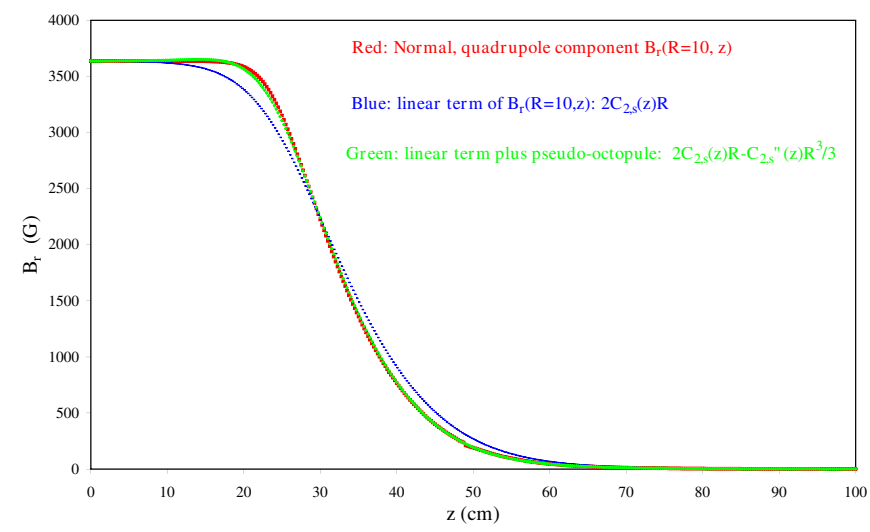

FIG. 21. (Color) Radial component $B_{r}(R=10, z)$ of $30 \mathrm{Q} 58$ and its constituents. 
pseudo-octupole. The pseudomultipoles are produced by magnetic fringe field. If $C_{2, s}$ has no $z$ dependence, its higher derivatives vanish, and the pseudomultipoles disappear. Figure 21 shows the radial component $B_{r}(R, z)$ of 30Q58 at $R=10 \mathrm{~cm}$ and its first two constituents: linear gradient and pseudo-octupole. It is clear that the linear term alone is not accurate for $B_{r}(R, z)$. A third-order representation, which includes the linear gradient and the pseudo-octupole only, is usually a good approximation of the real field.
The third-order aberrations of a quadrupole magnet with fringes have been studied before [7,9,10,19-21]. In this case, the particle optics can be expressed by

$$
\mathbf{x}_{2}=\mathbf{M} \mathbf{x}_{1}+\Delta \mathbf{x}
$$

where $\mathbf{x}_{1}$ and $\mathbf{x}_{2}$ are the phase space vectors for the $x$-motion at the entrance $z_{1}$ and exit $z_{2}, \mathbf{M}$ is the first-order transfer matrix obtained before, and $\Delta \mathbf{x}$ is the third-order aberration vector being found from

$$
\begin{aligned}
\Delta \mathbf{x}= & {\left[\begin{array}{llllllllll}
P_{10} & P_{11} & P_{12} & P_{13} & Q_{10} & Q_{11} & Q_{12} & R_{10} & R_{11} & R_{12} \\
P_{20} & P_{21} & P_{22} & P_{23} & Q_{20} & Q_{21} & Q_{22} & R_{20} & R_{21} & R_{22}
\end{array}\right] } \\
& \times\left[\begin{array}{llllllllll}
x_{1}^{3} & x_{1}^{2} x_{1}^{\prime} & x_{1} x_{1}^{\prime 2} & x_{1}^{\prime 3} & x_{1} y_{1}^{2} & x_{1} y_{1} y_{1}^{\prime} & x_{1} y_{1}^{\prime 2} & x_{1}^{\prime} y_{1}^{2} & x_{1}^{\prime} y_{1} y_{1}^{\prime} & x_{1}^{\prime} y_{1}^{\prime 2}
\end{array}\right]^{T} .
\end{aligned}
$$

The first term in the RHS of Eq. (A3) is the aberration coefficient matrix, while the second term is a vector for the initial conditions. There is a similar expression for the $y$-motion. Thus, there are 40 aberration coefficients,

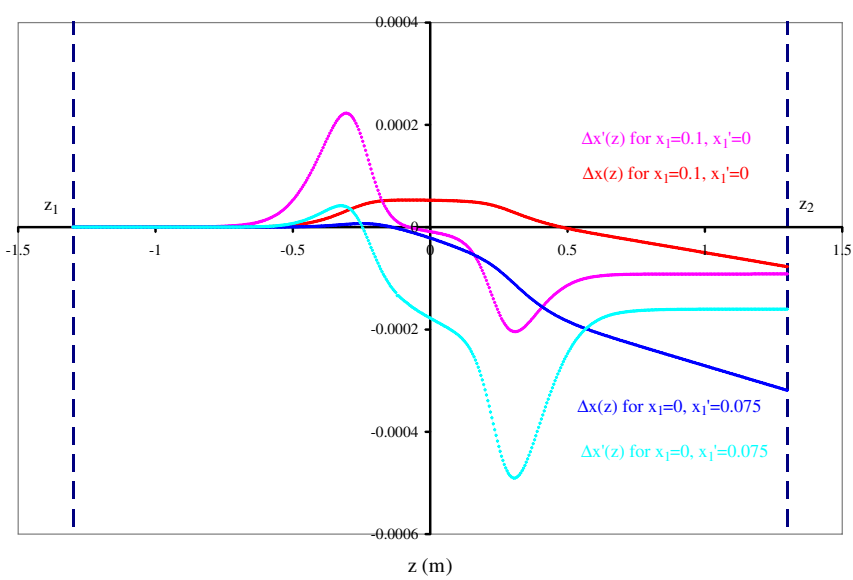

FIG. 22. (Color) Third-order aberrations on the $x$-plane in a focusing 30Q58. though not all independent due to the quadrupole symmetry. The third-order differential equations of motion can be obtained by employing the field expressions in Eq. (A1) and keeping all the dynamic variable terms up to a thirdorder:

$$
\begin{aligned}
x^{\prime \prime}+k x= & k\left(x^{\prime} y y^{\prime}-\frac{3}{2} x x^{\prime 2}-\frac{1}{2} x y^{\prime 2}\right)+k^{\prime} x y y^{\prime} \\
& +\frac{1}{12} k^{\prime \prime}\left(3 x y^{2}+x^{3}\right), \\
y^{\prime \prime}-k y= & -k\left(y^{\prime} x x^{\prime}-\frac{3}{2} y y^{\prime 2}-\frac{1}{2} y x^{\prime 2}\right)-k^{\prime} y x x^{\prime} \\
& -\frac{1}{12} k^{\prime \prime}\left(3 y x^{2}+y^{3}\right) .
\end{aligned}
$$

Equations (A4a) and (A4b) are usually solved by replacing $x, x^{\prime}, y, y^{\prime}$ on the right-hand side by the appropriate firstorder solutions. Here we only illustrate the third-order effects for particles on two symmetry planes and compare the results with the first-order theory. It can be shown that the third-order aberration vector taking into account $x_{1}{ }^{3}$ only $\left(P_{10}, P_{20}\right.$ terms) can be found from

TABLE VI. Numerical data for third-order effect in 30Q58.

\begin{tabular}{lcclcc}
\hline \hline & Focusing quad & & & Defocusing quad \\
\hline Initial $x_{1}(\mathrm{~m})$ & 0.1 & 0 & Initial $y_{1}(\mathrm{~m})$ & 0.1 & 0 \\
Initial $x_{1}{ }^{\prime}$ & 0 & 0.075 & Initial $y_{1}{ }^{\prime}$ & 0 & 0.075 \\
1st-order $x_{2}(\mathrm{~m})$ & 0.04611 & 0.14369 & 1st-order $y_{2}(\mathrm{~m})$ & 0.15924 & 0.25069 \\
1 st-order $x_{2}{ }^{\prime}$ & -0.04110 & 0.03458 & 1 st-order $y_{2}{ }^{\prime}$ & 0.04594 & 0.11943 \\
$\Delta x(\mathrm{~m})$ & $-7.73 \times 10^{-5}$ & $-3.19 \times 10^{-4}$ & $\Delta y(\mathrm{~m})$ & $-1.80 \times 10^{-5}$ & $6.95 \times 10^{-4}$ \\
$\Delta x^{\prime}$ & $-9.17 \times 10^{-5}$ & $-1.60 \times 10^{-4}$ & $\Delta y^{\prime}$ & $-5.40 \times 10^{-5}$ & $3.87 \times 10^{-4}$ \\
$\Delta x(\%)$ & $-0.17 \%$ & $-0.22 \%$ & $\Delta y(\%)$ & $-0.011 \%$ & $0.28 \%$ \\
$\Delta x^{\prime}(\%)$ & $0.22 \%$ & $-0.46 \%$ & $\Delta y^{\prime}(\%)$ & $-0.12 \%$ & $0.32 \%$ \\
$P_{10}\left(\mathrm{~m}^{-2}\right)$ & -0.0773 & & $P_{10}\left(\mathrm{~m}^{-2}\right)$ & -0.0180 & \\
$P_{20}\left(\mathrm{~m}^{-3}\right)$ & -0.0917 & & $P_{20}\left(\mathrm{~m}^{-3}\right)$ & -0.0540 & 1.647 \\
$P_{13}(\mathrm{~m})$ & & -0.756 & $P_{13}(\mathrm{~m})$ & & 0.917 \\
$P_{23}$ & & -0.379 & $P_{23}$ & & \\
\hline \hline
\end{tabular}




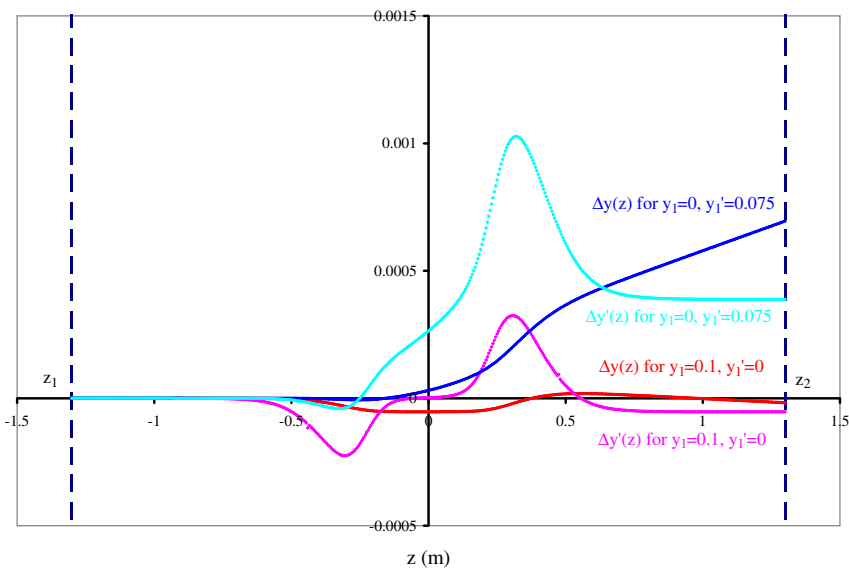

FIG. 23. (Color) Third-order aberrations on the $y$-plane in a defocusing 30Q58.

$$
\Delta x^{\prime \prime}+k \Delta x=\left(-\frac{3}{2} k M_{11} M_{21}^{2}+\frac{1}{12} k^{\prime \prime} M_{11}^{3}\right) x_{1}^{3} ;
$$

while the third-order aberration vector taking into account $x_{1}{ }^{3}$ only $\left(P_{13}, P_{23}\right.$ terms $)$ can be found from

$$
\Delta x^{\prime \prime}+k \Delta x=\left(-\frac{3}{2} k M_{12} M_{22}^{2}+\frac{1}{12} k^{\prime \prime} M_{12}^{3}\right) x_{1}^{\prime 3} .
$$

For the $y$-motion, the third-order aberrations for the two special rays can be found from the following equations:

$$
\begin{aligned}
& \Delta y^{\prime \prime}-k \Delta y=\left(\frac{3}{2} k M_{11} M_{21}^{2}-\frac{1}{12} k^{\prime \prime} M_{11}^{3}\right) y_{1}^{3}, \\
& \Delta y^{\prime \prime}-k \Delta y=\left(\frac{3}{2} k M_{12} M_{22}^{2}-\frac{1}{12} k^{\prime \prime} M_{12}^{3}\right) y_{1}^{\prime 3} .
\end{aligned}
$$

We first apply the theory to a single 30Q58. In Fig. 22 we plot the numerical solutions of Eqs. (A5a) and (A5b), which are the third-order aberrations on the $x$-plane through a focusing 30Q58. The corresponding first-order rays result from two cases: one with the initial condition of

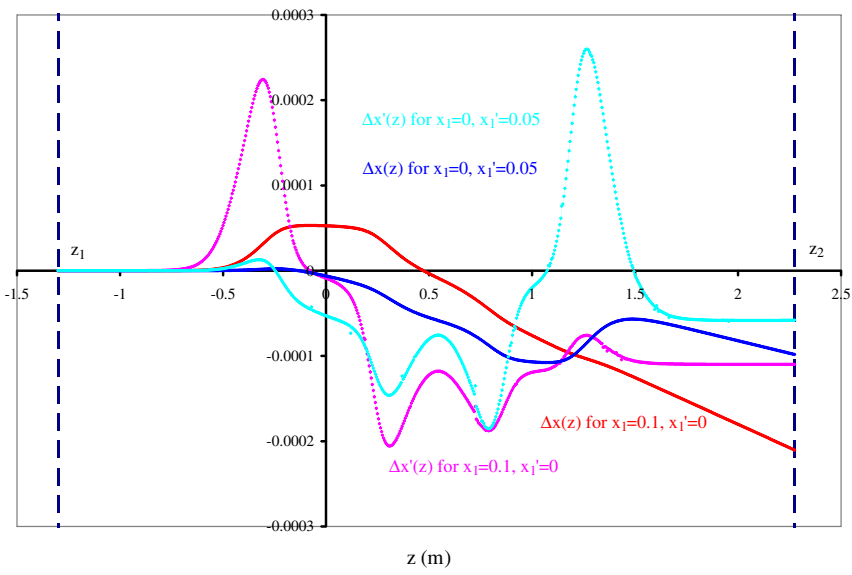

FIG. 24. (Color) Third-order aberrations on the $x$-plane through quadrupole doublet assembly.

$x_{1}=0.1 \mathrm{~m}$ and $x_{1}{ }^{\prime}=0$ and the other with $x_{1}=0$ and $x_{1}{ }^{\prime}=0.075$. The computed particle parameters and the aberration coefficients are listed in Table VI, where $\Delta x(\%)$ and $\Delta x^{\prime}(\%)$ are relative changes with respect to the linear optics parameters at the exit $z_{2}=1.3 \mathrm{~m}$. For the first ray, the nonlinear field changes $x_{2}$ and $x_{2}{ }^{\prime}$ by $-0.17 \%$ and $0.22 \%$, respectively; while for the second ray the changes in $x_{2}$ and $x_{2}{ }^{\prime}$ are $-0.22 \%$ and $-0.46 \%$, respectively. Similarly, the third-order aberrations on the $y$-plane through a defocusing 30Q58 are solved by Eqs. (A6a) and (A6b), as plotted in Fig. 23 and also listed in Table VI.

The third-order analyses for a single 30Q44 have been done in the same way, and the numerical results are listed in Table VII.

The third-order aberrations in the SNS quadrupole doublet assembly can be calculated in the same way as well. Here we employ Eqs. (A5a) and (A5b) to compute the third-order aberrations $\left[\Delta x(z), \Delta x^{\prime}(z)\right]$ on the $x$-plane due to the aberration coefficients $P_{0}$ and $P_{3}$. The corresponding two first-order rays are the one with $x_{1}=0.1 \mathrm{~m}$ and $x_{1}{ }^{\prime}=$ 0 , and the other with $x_{1}=0$ and $x_{1}{ }^{\prime}=0.05$, respectively.

TABLE VII. 30Q44 third-order aberration parameters.

\begin{tabular}{lcclcc}
\hline \hline & Defocusing quad & & \multicolumn{3}{c}{ Focusing quad } \\
\hline Initial $x_{1}(\mathrm{~m})$ & 0.1 & 0 & Initial $y_{1}(\mathrm{~m})$ & 0.1 & 0 \\
Initial $x_{1}{ }^{\prime}$ & 0 & 0.075 & Initial $y_{1}{ }^{\prime}$ & 0 & 0.075 \\
1st-order $x_{2}(\mathrm{~m})$ & 0.0558 & 0.1469 & 1 st-order $y_{2}(\mathrm{~m})$ & 0.1474 & 0.2307 \\
1 st-order $x_{2}{ }^{\prime}$ & -0.0352 & 0.0418 & 1 st-order $y_{2}{ }^{\prime}$ & 0.0381 & 0.1105 \\
$\Delta x(\mathrm{~m})$ & $-6.30 \times 10^{-5}$ & $-2.73 \times 10^{-4}$ & $\Delta y(\mathrm{~m})$ & $-2.78 \times 10^{-5}$ & $4.91 \times 10^{-4}$ \\
$\Delta x^{\prime}$ & $-7.49 \times 10^{-5}$ & $-1.45 \times 10^{-4}$ & $\Delta y^{\prime}$ & $-5.09 \times 10^{-5}$ & $2.78 \times 10^{-4}$ \\
$\Delta x(\%)$ & $-0.11 \%$ & $-0.19 \%$ & $\Delta y(\%)$ & $-0.019 \%$ & $0.21 \%$ \\
$\Delta x^{\prime}(\%)$ & $0.21 \%$ & $-0.35 \%$ & $\Delta y^{\prime}(\%)$ & $-0.13 \%$ & $0.25 \%$ \\
$P_{10}\left(\mathrm{~m}^{-2}\right)$ & -0.0630 & & $P_{10}\left(\mathrm{~m}^{-2}\right)$ & -0.0278 & \\
$P_{20}\left(\mathrm{~m}^{-3}\right)$ & -0.0749 & & $P_{20}\left(\mathrm{~m}^{-3}\right)$ & -0.0509 & 1.164 \\
$P_{13}(\mathrm{~m})$ & & -0.647 & $P_{13}(\mathrm{~m})$ & & 0.658 \\
$P_{23}$ & & -0.343 & $P_{23}$ & & \\
\hline \hline
\end{tabular}




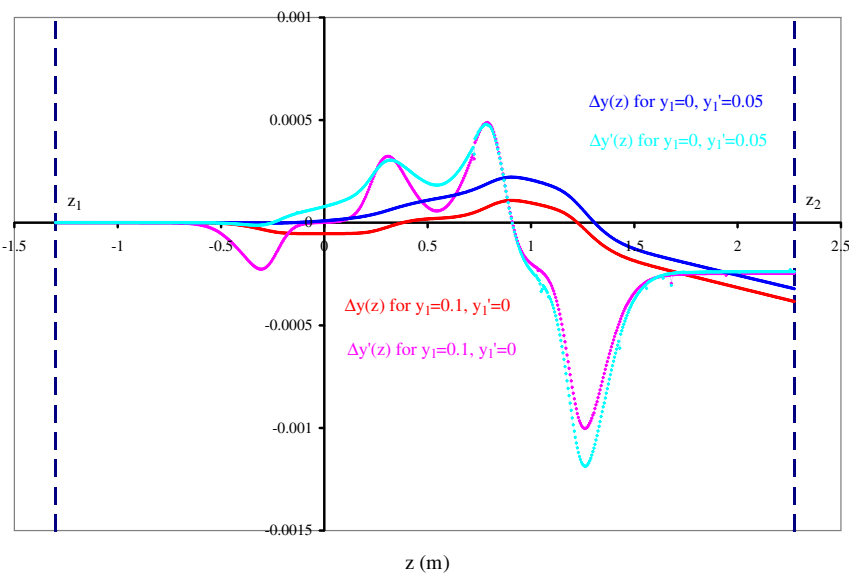

FIG. 25. (Color) Third-order aberrations on the $y$-plane through quadrupole doublet assembly.

The results are plotted in Fig. 24. At $z_{2}=2.275 \mathrm{~m}, \Delta x=$ $-2.10 \times 10^{-04} \mathrm{~m}, \Delta x^{\prime}=-1.10 \times 10^{-04}$ due to $P_{0}$, and $\Delta x=-9.83 \times 10^{-05} \mathrm{~m}, \Delta x^{\prime}=-5.81 \times 10^{-05}$ due to $P_{3}$. They represent relative changes of $-0.61 \%$, $-0.58 \%,-0.061 \%$, and $-0.10 \%$, respectively, in comparison with the first-order matrix solutions which are the elements in $\mathbf{M}_{x}$ in (20) scaled by the initial parameters. Similarly, we use Eqs. (A6a) and (A6b) to calculate the third-order aberrations on the $y$-plane due to the aberration coefficients $P_{0}$ and $P_{3}$. The results are plotted in Fig. 25. At $z_{2}=2.275 \mathrm{~m}, \quad \Delta y=-3.84 \times 10^{-04} \mathrm{~m}, \quad \Delta y^{\prime}=$ $-2.47 \times 10^{-04}$ due to $P_{0}$, and $\Delta y=-3.22 \times 10^{-04} \mathrm{~m}$, and $\Delta y^{\prime}=-2.39 \times 10^{-04}$ due to $P_{3}$. They represent relative changes of $-0.28 \%, 4.20 \%,-0.18 \%$, and $-0.85 \%$, respectively, from the first-order results.

[1] J. Wei, D. T. Abell, J. Beebe-Wang, M. Blaskiewicz, P. R. Cameron, N. Catalan-Lasheras, G. Danby, A. V. Fedotov, C. Gardner, J. Jackson, Y. Y. Lee, H. Ludewig, N. Malitsky, W. Meng, Y. Papaphilippou, D. Raparia, N. Tsoupas, W. T. Weng, R. L. Witkover, and S. Y. Zhang, Phys. Rev. ST Accel. Beams 3, 080101 (2000).

[2] N. Tsoupas, J. Brodowski, W. Meng, J. Wei, Y. Y. Lee, and J. Tuozzolo, in Proceedings of the 2002 European Particle Accelerator Conference, Paris (EPS, Paris, 2002), p. 1106.

[3] N. Tsoupas, J. Jackson, Y. Y. Lee, D. Raparia, and J. Wei, in Proceedings of the 2003 Particle Accelerator Conference, Portland, Oregon, p. 2153.

[4] H. Wollnik and H. Ewald, Nucl. Instrum. Methods 36, 93 (1965).

[5] H. Hubner and H. Wollnik, Nucl. Instrum. Methods 86, 141 (1970).

[6] G. E. Lee-Whiting, Nucl. Instrum. Methods 76, 305 (1969).

[7] G. E. Lee-Whiting, Nucl. Instrum. Methods 83, 232 (1970).
[8] H. Matsuda and H. Wollnik, Nucl. Instrum. Methods 77, 283 (1970).

[9] H. Matsuda and H. Wollnik, Nucl. Instrum. Methods 103, 117 (1972).

[10] M. Venturini, in Proceedings of the 1999 Particle Accelerator Conference, New York (IEEE, New York, 1999), p. 1590.

[11] M. Berz, B. Erdelyi, and K. Makino, Phys. Rev. ST Accel. Beams 3, 124001 (2000).

[12] S. Bernal, H. Li, R. A. Kishek, B. Quinn, M. Walter, M. Reiser, and P. G. O'Shea, C. K. Allen, Phys. Rev. ST Accel. Beams 9, 064202 (2006).

[13] J. G. Wang, N. Tsoupas, and M. Venturini, in Proceedings of the 2005 Particle Accelerator Conference, Knoxville, TN, p. 3865; also see J. G. Wang, SNS-NOTE-MAG-154, 2005.

[14] OPERA-3d (an OPerating environment for Electromagnetic Research and Analysis) is the preprocessing and postprocessing system for electromagnetic analysis programs such as TOSCA (for nonlinear magnetostatic or electrostatic field and current flow problems) developed by Vector Fields Limited, England.

[15] A. K. Jain, in Proceedings of CERN Accelerator School on Measurement and Alignment of Accelerator and Detector Magnets, 1997, Anacapri, Italy.

[16] K. Halbach, LBL Engineering Note, 1972.

[17] J.G. Wang, Phys. Rev. ST Accel. Beams 9, 012401 (2006).

[18] M. Venturini and A. Dragt, Nucl. Instrum. Methods Phys. Res., Sect. A 427, 387 (1999).

[19] K. G. Steffen, High Energy Beam Optics (Interscience Publishers, New York, 1964).

[20] P. Grivet, Electron Optics (Pergamon Press, Oxford, 1965).

[21] P.W. Hawkes, Quadrupole Optics (Springer-Verlag, Heidelberg, 1966).

[22] H. Wollnik, Optics of Charged Particles (Academic Press, Inc., San Diego, 1987).

[23] D.C. Carey, The Optics of Charged Particle Beams (Harwood Academic Publishers, Paris, 1987).

[24] M. Reiser, Theory and Design of Charged Particle Beams (John Wiley \& Sons, Inc., New York, 1994).

[25] Stephen Wolfram, The MATHEMATICA Book (Wolfram Media/Cambridge University Press, Cambridge, New York, and Melbourne, 1999), 4th ed.

[26] D. C. Carey, K. L. Brown, and F. Rothacker, SLAC-R-530, Fermilab-pub-98-310, UC-414, 1998.

[27] H. Grote and F. C. Iselin, CERN/SL/90-13 (AP), 1990.

[28] H.J. Stein (private communication); also see CERN Courier, 1993, pp. 3-4.

[29] Y. Papaphilippou, Y. Y. Lee, and W. Meng, in Proceedings of the 2001 Particle Accelerator Conference, Chicago, p. 1667.

[30] D. Raparia, in ASAC (Accelerator System Advisory Committee) Review of the SNS Project, 2004.

[31] P. M. Morse and H. Feshbach, Methods of Theoretical Physics (McGraw-Hill, New York, 1953). 